\title{
Packing and Conformational Polymorphism in 1,2-Bis(amino carbonyl-(1-tert-butyl-1 H-pyrazole-(3)5-yl))-ethanes. Illuminating Examples of Highly Flexible Molecules
}

\author{
Geórgia C. Zimmer ${ }^{\dagger}$, Anderson B. Pagliari ${ }^{\dagger}$, Vanessa B. Solner ${ }^{\dagger}$, Manfredo Hörner ${ }^{\dagger}$, Helio G. \\ Bonacorso $^{\dagger}$, Nilo Zanatta ${ }^{\dagger}$, Marcos A. P. Martins ${ }^{\dagger *}$
}

\begin{abstract}
†Núcleo de Química de Heterociclos (NUQUIMHE), Department of Chemistry, Federal University of Santa Maria (UFSM), 97105-900, Santa Maria, RS, Brazil

*E-mail of corresponding author: marcos.nuquimhe@gmail.com
\end{abstract}

\section{Table of Contents}

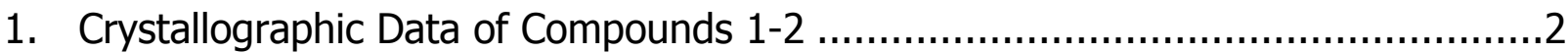

2. Supramolecular Cluster..............................................................................

2.1 Molecular Coordination Number and Supramolecular Cluster ..........................5

2.2 Topological and energetic data of compounds $1-2$...................................10

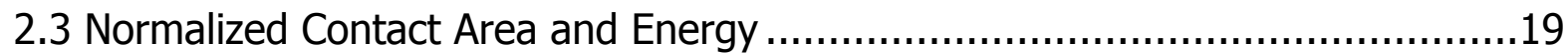

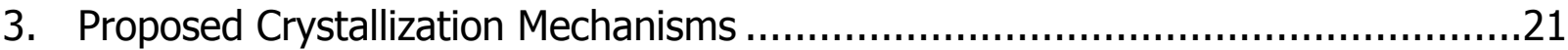

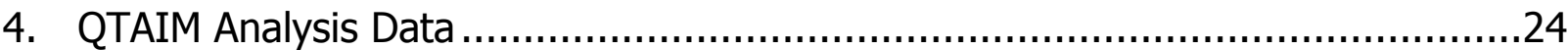




\section{Crystallographic Data of Compounds 1-2}

Table S1. X-ray data for compounds 1a-b

\begin{tabular}{|c|c|c|c|}
\hline Compound & 1aI & 1aII & 1b \\
\hline CCDC & 2063650 & 2063637 & 1453941 \\
\hline Empirical formula & $\mathrm{C}_{20} \mathrm{H}_{32} \mathrm{~N}_{6} \mathrm{O}_{2}$ & $\mathrm{C}_{20} \mathrm{H}_{32} \mathrm{~N}_{6} \mathrm{O}_{2}$ & $\mathrm{C}_{30} \mathrm{H}_{34} \mathrm{~F}_{2} \mathrm{~N}_{6} \mathrm{O}_{2} \cdot 2 \mathrm{H}_{2} \mathrm{O}$ \\
\hline $\mathrm{Mw}$ & 88.52 & 388.52 & 584.66 \\
\hline$T[\mathrm{~K}]$ & 100 & 100 & 293 \\
\hline Crystal system & Monoclinic & Tricline & Triclinc \\
\hline Space group & $P 2_{1} / c$ & $P-1$ & $P-1$ \\
\hline$\lambda(\AA)$ & Mo / 0.71073 & Mo / 0.71073 & Mo / 0.71073 \\
\hline$a[\AA]$ & $6.207(3)$ & $6.3362(2)$ & $8.0475(3)$ \\
\hline$b[\AA]$ & $11.186(6)$ & $7.9693(3)$ & $9.0924(5)$ \\
\hline$c[\AA]$ & $15.526(8)$ & $10.7737(4)$ & $11.3156(5)$ \\
\hline$a\left[^{\circ}\right]$ & 90 & $87.1720(10)$ & $108.716(3)$ \\
\hline$\beta\left[^{\circ}\right]$ & $92.969(13)$ & $83.2190(10)$ & $95.044(2)$ \\
\hline$y\left[^{\circ}\right]$ & 90 & $87.6340(10)$ & $95.755(2)$ \\
\hline$V\left[\AA^{3}\right]$ & $1076.4(10)$ & $539.21(3)$ & $773.88(6)$ \\
\hline Z & $4(0.5)$ & $1(0.5)$ & $2(0.5)$ \\
\hline$D_{\text {calcd. }}\left[\mathrm{g} \mathrm{cm}^{-3}\right]$ & 1.199 & 1.196 & 1.255 \\
\hline$\mu\left[\mathrm{mm}^{-1}\right]$ & 0.080 & 0.080 & 0.093 \\
\hline F(000) & 420 & 210 & 310 \\
\hline Crystal size (mm) & $0.40 \times 0.20 \times 0.19$ & $0.30 \times 0.25 \times 0.20$ & $0.62 \times 0.56 \times 0.37$ \\
\hline$\theta$ range for data & 3.87 to 30.09 & 3.24 to 27.14 & 1.92 to 27.27 \\
\hline Collection (deg) h. k. I & $-8 \leq h \leq 8$ & $-8 \leq h \leq 8$ & $-10 \leq h \leq 10$ \\
\hline range & $-15 \leq k \leq 15$ & $-10 \leq k \leq 10$ & $-11 \leq k \leq 11$ \\
\hline & $-21 \leq / \leq 21$ & $-13 \leq / \leq 10$ & $-14 \leq / \leq 14$ \\
\hline Reflections collected/unique & $14140 / 3121$ & 17455 / 2357 & 22264 / 3466 \\
\hline 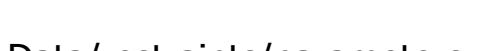 & t & {$[R($ Int $)=$} & {$[R($ Int $)=0.0252]$} \\
\hline ameters & $3121 / 0 / 127$ & $2357 /$ 18/ 131 & $3466 / 4 / 189$ \\
\hline Absoption correction & Multi-scan & Multi-scan & Gaussian \\
\hline Refinement method & $\begin{array}{l}\text { Full-matrix least- } \\
\text { squares on } \mathrm{F}^{\wedge} 2\end{array}$ & $\begin{array}{l}\text { Full-matrix least- } \\
\text { squares on } \mathrm{F}^{\wedge} 2\end{array}$ & $\begin{array}{l}\text { Full-matrix least- } \\
\text { squares on } \mathrm{F}^{\wedge} 2\end{array}$ \\
\hline Final $R$ indices & $\mathrm{R} 1=0.0713$. & $\mathrm{R} 1=0.0930$. & $\mathrm{R} 1=0.0517$ \\
\hline & $\mathrm{wR2}=0.2025$ & $\mathrm{wR2}=0.2450$ & $\mathrm{wR2}=0.1332$ \\
\hline$R$ all data & $R 1=0.0959$. & $\mathrm{R} 1=0.1027$ & $\mathrm{R} 1=0.0669$. \\
\hline & $\mathrm{WR2}=0.2219$ & $\mathrm{WR2}=0.253 \mathrm{I}$ & $\begin{array}{l}W R 2=0.1466 \\
1039\end{array}$ \\
\hline & 1.043 & 1.053 & 1.039 \\
\hline $\begin{array}{l}\text { Largest diff. peak and hole } \\
\left(e \AA^{3}\right)\end{array}$ & 0.817 and -0.299 & 1.012 and -0.488 & 0.509 and -0.405 \\
\hline
\end{tabular}


Table S2. X-ray data for compounds $\mathbf{1 c - d}$ and $\mathbf{2 a}$

\begin{tabular}{|c|c|c|c|c|}
\hline Compound & 1c & 1d & 2aI & 2aII \\
\hline CCDC & 2063652 & 2063657 & 2063664 & 2063665 \\
\hline Empirical formula & $\mathrm{C}_{30} \mathrm{H}_{34} \mathrm{Cl}_{2} \mathrm{~N}_{6} \mathrm{O}_{2}$ & $\begin{array}{l}\mathrm{C}_{30} \mathrm{H}_{34} \mathrm{Br}_{2} \mathrm{~N}_{6} \mathrm{O}_{2} . \\
2\left(\mathrm{C}_{2} \mathrm{H}_{3} \mathrm{CN}\right)\end{array}$ & $\mathrm{C}_{20} \mathrm{H}_{32} \mathrm{~N}_{6} \mathrm{O}_{2}$ & $\begin{array}{l}\mathrm{C}_{20} \mathrm{H}_{32} \mathrm{~N}_{6} \mathrm{O}_{2} . \\
2\left(\mathrm{H}_{2} \mathrm{O}\right)\end{array}$ \\
\hline $\mathrm{Mw}$ & 581.53 & 752.56 & 388.51 & 424.55 \\
\hline$T[\mathrm{~K}]$ & 100 & $296(2)$ & 100 & 293 \\
\hline Crystal system & Monoclinic & Monoclinic & Monoclinic & Monoclinic \\
\hline Space group & $P 2_{1} / n$ & $\mathrm{C} 2 / \mathrm{c}$ & $P 2_{1} / C$ & $P 2_{1} / C$ \\
\hline$\lambda(\AA)$ & Mo / 0.71073 & Cu / 1.54178 & Mo / 0.71073 & $\mathrm{Cu} / 1.54178$ \\
\hline$a[\AA]$ & $6.2058(6)$ & $18.2905(13)$ & $9.6598(5)$ & $9.4612(2)$ \\
\hline$b[\AA]$ & $7.4087(8)$ & $8.4837(5)$ & $26.0820(15)$ & $18.6670(4)$ \\
\hline$c[\AA]$ & $31.068(3)$ & $24.6711(15)$ & $9.6981(5)$ & $14.2833(3)$ \\
\hline$a\left[^{\circ}\right]$ & $90.000(3)$ & 90 & 90 & 90 \\
\hline$\beta\left[^{\circ}\right]$ & 89.033 (3) & $110.526(6)$ & 117.691 & $101.4630(10)$ \\
\hline$V\left[^{\circ}\right]$ & $90.000(3)$ & 90 & 90 & 90 \\
\hline$V\left[\AA^{3}\right]$ & $1428.3(3)$ & $3585.2(4)$ & $2163.6(2)$ & $2472.29(9)$ \\
\hline Z & $2(0.5)$ & $4(0.5)$ & $4(1)$ & $4(1)$ \\
\hline$D_{\text {calcd. }}\left[\mathrm{g} \mathrm{cm}^{-3}\right]$ & 1.352 & 1.394 & 1.193 & 1.141 \\
\hline$\mu\left[\mathrm{mm}^{-1}\right]$ & 0.267 & 3.199 & 0.080 & 0.659 \\
\hline F(000) & 612 & 1544 & 840 & 920 \\
\hline Crystal size (mm) & $0.31 \times 0.05 \times 0.05$ & $0.16 \times 0.14 \times 0.06$ & $0.28 \times 0.15 \times 0.09$ & $0.44 \times 0.26 \times 0.18$ \\
\hline$\theta$ range for data & 2.623 to 27.15 & 3.826 to 68.634 & 2.381 to 27.145 & 4.769 to 68.261 \\
\hline Collection (deg) $h$. & $-7 \leq h \leq 7$ & $-17 \leq h \leq 21$ & $-12 \leq h \leq 12$ & $-11 \leq h \leq 11$ \\
\hline$k$. /range & $-9 \leq k \leq 9$ & $-10 \leq k \leq 10$ & $-33 \leq k \leq 33$ & $-22 \leq k \leq 22$ \\
\hline & $-39 \leq / \leq 39$ & $-29 \leq / \leq 29$ & $-12 \leq / \leq 12$ & $-17 \leq / \leq 17$ \\
\hline Reflections & 37699 / 3150 & 26365 / 3232 & $31440 / 4782$ & 29580 / 4434 \\
\hline collected/unique & {$[R($ int $)=0.198]$} & {$[R($ int $)=0.098]$} & {$[R($ int $)=0.065]$} & {$[R($ int $)=0.027]$} \\
\hline $\begin{array}{l}\text { Data/restraints/par } \\
\text { a-meters }\end{array}$ & 3150 / 0 / 181 & 3232 / 0/ 209 & 4782 / 0 / 289 & 4434 / 0 / 295 \\
\hline Absoption & Multi-scan & Multi-scan & Multi-scan & Multi-scan \\
\hline $\begin{array}{l}\text { correction } \\
\text { Refinement method }\end{array}$ & $\begin{array}{l}\text { Full-matrix least- } \\
\text { squares on } \mathrm{F}^{\wedge} 2\end{array}$ & $\begin{array}{l}\text { Full-matrix least- } \\
\text { squares on } \mathrm{F}^{\wedge} 2\end{array}$ & $\begin{array}{l}\text { Full-matrix least- } \\
\text { squares on } \mathrm{F}^{\wedge} 2\end{array}$ & $\begin{array}{l}\text { Full-matrix least- } \\
\text { squares on } F^{\wedge} 2\end{array}$ \\
\hline Final $R$ indices & $\begin{array}{l}R 1=0.0849 \\
W R 2=0.1634\end{array}$ & $\begin{array}{l}\mathrm{R} 1=0.0602 . \\
\mathrm{WR2}=0.1501\end{array}$ & $\begin{array}{l}R 1=0.0595 . \\
W R 2=0.1112\end{array}$ & $\begin{array}{l}R 1=0.0506 . \\
w R 2=0.1384\end{array}$ \\
\hline$R$ all data & $\begin{array}{l}R 1=0.1528 \\
W R 2=0.1814\end{array}$ & $\begin{array}{l}R 1=0.0917 \\
W R 2=0.1637\end{array}$ & $\begin{array}{l}\mathrm{R} 1=0.0970 . w R 2 \\
=0.1230\end{array}$ & $\begin{array}{l}R 1=0.0600 . \\
w R 2=0.1469\end{array}$ \\
\hline $\begin{array}{l}\text { Goodness of fit on } \\
R\end{array}$ & 1.165 & 1.063 & 1.060 & 1.045 \\
\hline $\begin{array}{l}\text { Largest diff. peak } \\
\text { and hole }\left(e \AA^{3}\right)\end{array}$ & 0.502 and -0.397 & 0.338 and -0.551 & 0.361 and -0.228 & 0.352 and -0.178 \\
\hline
\end{tabular}


Table S3. X-ray data for compounds $\mathbf{2 b}$-d.

\begin{tabular}{|c|c|c|c|c|}
\hline Compound & 2b & $2 c$ & 2dI & 2dII \\
\hline CCDC & 1453942 & 1498649 & 1498643 & 2063666 \\
\hline Empirical formula & $\mathrm{C}_{30} \mathrm{H}_{34} \mathrm{~F}_{2} \mathrm{~N}_{6} \mathrm{O}_{2}$ & $\mathrm{C}_{30} \mathrm{H}_{34} \mathrm{Cl}_{2} \mathrm{~N}_{6} \mathrm{O}_{2}$ & $\mathrm{C}_{30} \mathrm{H}_{34} \mathrm{Br}_{2} \mathrm{~N}_{6} \mathrm{O}_{2}$ & $\mathrm{C}_{30} \mathrm{H}_{34} \mathrm{Br}_{2} \mathrm{~N}_{6} \mathrm{O}_{2}$ \\
\hline Mw & 548.63 & 581.53 & 670.45 & 670.45 \\
\hline$T[\mathrm{~K}]$ & $293(2)$ & 293 & 293 & 296 \\
\hline Crystal system & Monoclinic & Monoclinic & Monoclinic & Tetragonal \\
\hline Space group & $C 2 / C$ & $C 2 / c$ & $C 2 / c$ & $P 4_{2} / \mathrm{n}$ \\
\hline$\lambda(\AA)$ & Mo / 0.71073 & Mo / 0.71073 & Mo / 0.71073 & $\mathrm{Cu} / 1.54178$ \\
\hline$a[\AA]$ & $25.2656(6)$ & $26.6417(8)$ & $27.6305(9)$ & $18.7994(4)$ \\
\hline$b[\AA]$ & $11.4447(2)$ & $11.3041(3)$ & $11.0991(3)$ & $18.7994(4)$ \\
\hline$c[\AA]$ & $9.7315(2)$ & $9.7939(2)$ & $9.8477(2)$ & $18.8804(6)$ \\
\hline$a\left[^{\circ}\right]$ & 90 & 90 & 90 & 90 \\
\hline$\beta\left[^{\circ}\right]$ & $94.7990(10)$ & $91.318(2)$ & $90.520(2)$ & 90 \\
\hline$V\left[^{\circ}\right]$ & 90 & 90 & 90 & 90 \\
\hline$V\left[\AA^{3}\right]$ & $2804.07(10)$ & $2948.76(13)$ & $3019.91(14)$ & 6672.7 \\
\hline$Z$ & $4(0.5)$ & $8(0.5)$ & $4(0.5)$ & $8(0.5)$ \\
\hline$D_{\text {calcd. }}\left[\mathrm{g} \mathrm{cm}^{-3}\right]$ & 1.300 & 1.310 & 1.475 & 1.335 \\
\hline$\mu\left[\mathrm{mm}^{-1}\right]$ & 0.093 & 0.258 & 2.722 & 3.353 \\
\hline F(000) & 1160 & 1224 & 1368 & 2736 \\
\hline Crystal size $(\mathrm{mm})$ & $0.44 \times 0.38 \times 0.20$ & $0.77 \times 0.54 \times 0.44$ & $0.45 \times 0.367 \times 0.27$ & $0.30 \times 0.21 \times 0.13$ \\
\hline$\theta$ range for data & 1.62 to 27.18 & 1.96 to 27.57 & 1.47 to 28.36 & 3.317 to 68.38 \\
\hline Collection (deg) h. k. I & $-32 \leq h \leq 32$ & $-34 \leq h \leq 34$ & $-36 \leq h \leq 36$ & $-22 \leq h \leq 22$ \\
\hline range & $-14 \leq k \leq 14$ & $-14 \leq k \leq 14$ & $-14 \leq k \leq 14$ & $-22 \leq k \leq 22$ \\
\hline & $-12 \leq / \leq 12$ & $-12 \leq / \leq 12$ & $-13 \leq / \leq 13$ & $-22 \leq / \leq 22$ \\
\hline $\begin{array}{l}\text { Reflections } \\
\text { collected/unique }\end{array}$ & $\begin{array}{l}38626 / 3119 \\
{[R(\text { int })=0.043]}\end{array}$ & $\begin{array}{l}40005 / 3402 \\
{[R \text { (int) }=00275]}\end{array}$ & $\begin{array}{l}34371 / 3758 \\
\text { R(int) = } 02971\end{array}$ & 102040 / 6111 \\
\hline $\begin{array}{l}\text { Data/restraints/paramet } \\
\text { ers }\end{array}$ & 3119 / 0/ 194 & $3402 / 0 / 181$ & 3758 / 0 / 182 & $6111 / 0 / 361$ \\
\hline Absoption correction & Gaussian & Gaussian & Gaussian & Multi-scan \\
\hline Refinement method & $\begin{array}{l}\text { Full-matrix least- } \\
\text { squares on } \mathrm{F}^{\wedge} 2\end{array}$ & $\begin{array}{l}\text { Full-matrix least- } \\
\text { squares on } \mathrm{F}^{\wedge} 2\end{array}$ & $\begin{array}{l}\text { Full-matrix least- } \\
\text { squares on } \mathrm{F}^{\wedge} 2\end{array}$ & $\begin{array}{l}\text { Full-matrix least- } \\
\text { squares on } \mathrm{F}^{\wedge} 2\end{array}$ \\
\hline Final $R$ indices & $\begin{array}{l}R 1=0.0399 \\
w R 2=0.0990\end{array}$ & $\begin{array}{l}R 1=0.0387 \\
W R 2=0.1026\end{array}$ & $\begin{array}{l}R 1=0.0340 \\
w R 2=0.0967\end{array}$ & $\begin{array}{l}R 1=0.0553 \\
w R 2=0.1679\end{array}$ \\
\hline$R$ all data & $\begin{array}{l}R 1=0.0649 \\
W R 2=0.1133\end{array}$ & $\begin{array}{l}R 1=0.0484 \\
W R 2=0.1097\end{array}$ & $\begin{array}{l}\mathrm{R} 1=0.0571 \\
\mathrm{WR} 2=0.1138\end{array}$ & $\begin{array}{l}R 1=0.0727 \\
W R 2=0.1831\end{array}$ \\
\hline Goodness of fit on $P$ & 1.039 & 1.052 & 1.111 & 1.044 \\
\hline $\begin{array}{l}\text { Largest diff. peak and } \\
\text { hole }\left(e \AA^{3}\right)\end{array}$ & 0.177 and -0.130 & 0.185 and -0.254 & 0.471 and -0.262 & 0.560 and -0.568 \\
\hline
\end{tabular}


Table S4. Characterization of dihedral angles for compounds 1-2. ${ }^{\text {a }}$

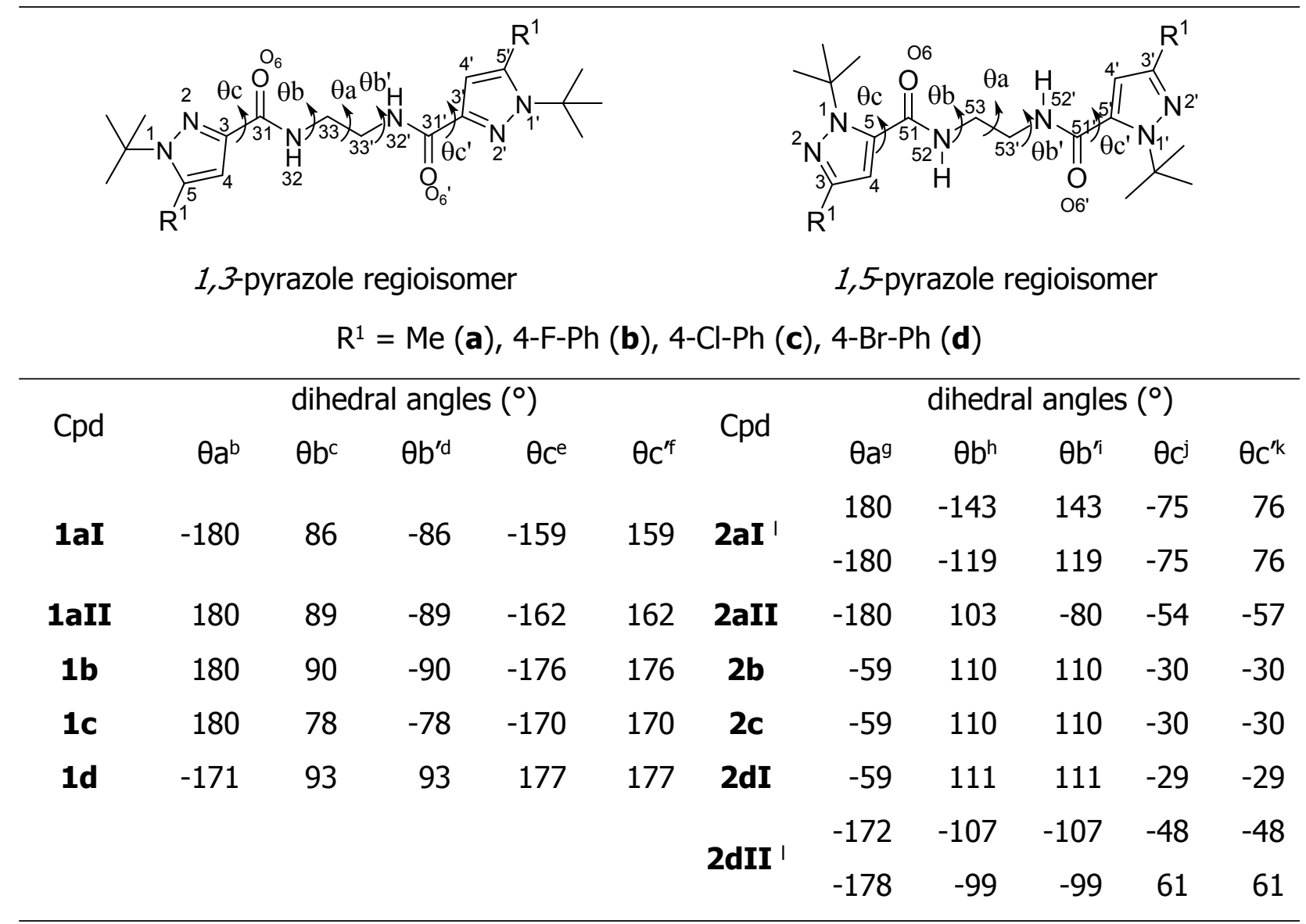

The negative sign refers to the definition of the dihedral angle counterclockwise and positive clockwise. bN32-C33-C33'-N32'; ' 'C31-N32-C33-C33'; ' 'C31'-N32'-C33'-C33; 'CC4-C3-C31-N32; fC4'-C3'-C31'-N32'; 9N52-C53-C53'-N52'; ' $\mathrm{C} 51-\mathrm{N} 52-\mathrm{C} 53-\mathrm{C} 53$ '; 'C51'-N52'-C53'-C53; iC4-C5-C51N52; ${ }^{k} \mathrm{C} 4-\mathrm{C} 5-\mathrm{C} 51-\mathrm{N} 52 ;{ }^{\prime} \mathrm{Z}^{\prime}=1$ (two half molecules).

\section{Supramolecular Cluster}

\subsection{Molecular Coordination Number and Supramolecular Cluster}

Table S5. Molecular Coordination Number (MCN) of compounds 1-2.

\begin{tabular}{cccc}
\hline Compound & $M C N$ & Compound & $M C N$ \\
$\mathbf{1 a I}$ & 18 & $\mathbf{2 a I}$ & 14 \\
$\mathbf{1 a I I}$ & 16 & $\mathbf{2 a I I}$ & $22\left(14: 8 \mathrm{H}_{2} \mathrm{O}\right)$ \\
$\mathbf{1 b}$ & $24(14: 10)$ & $\mathbf{2 b}$ & 16 \\
$\mathbf{1 c}$ & 16 & $\mathbf{2 c}$ & 16 \\
$\mathbf{1 d}$ & 14 & $\mathbf{2 d I}$ & 16 \\
& & $\mathbf{2 d I I}$ & 20 \\
\hline
\end{tabular}


Table S6. Supramolecular clusters and molecules labels of compounds 1-2.

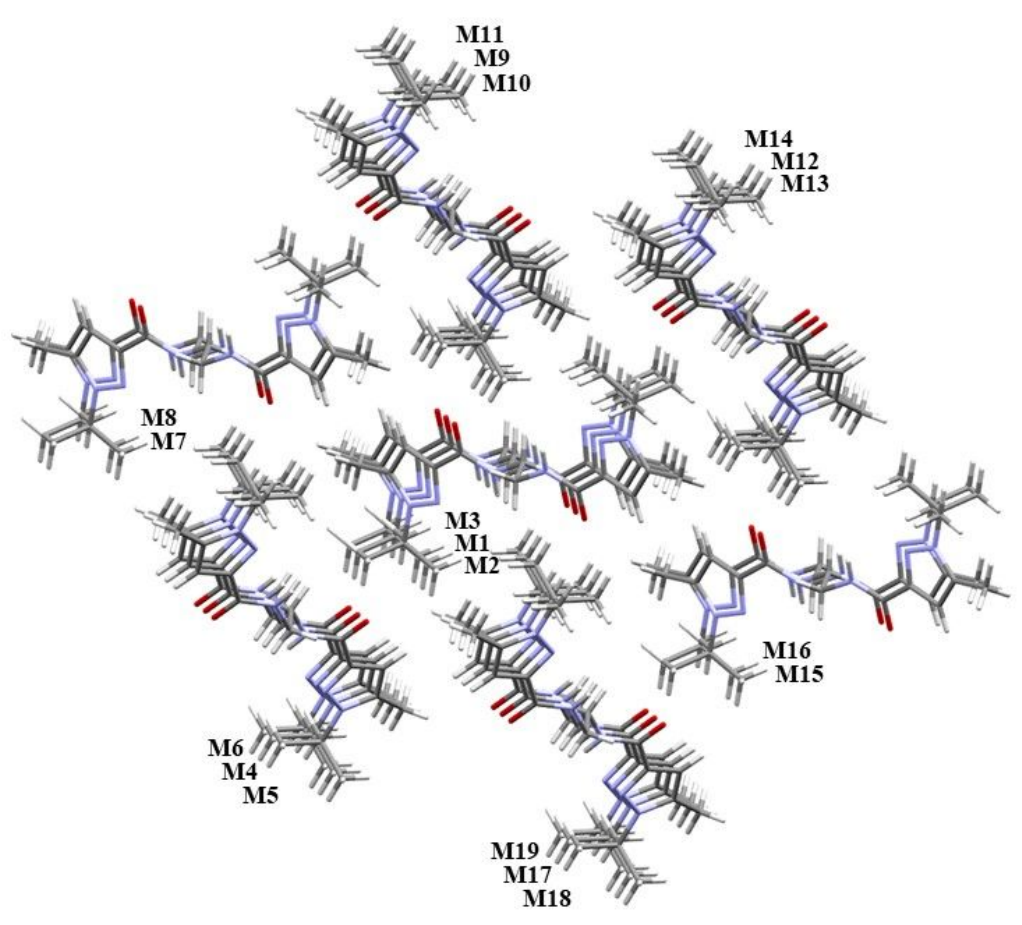

1aI

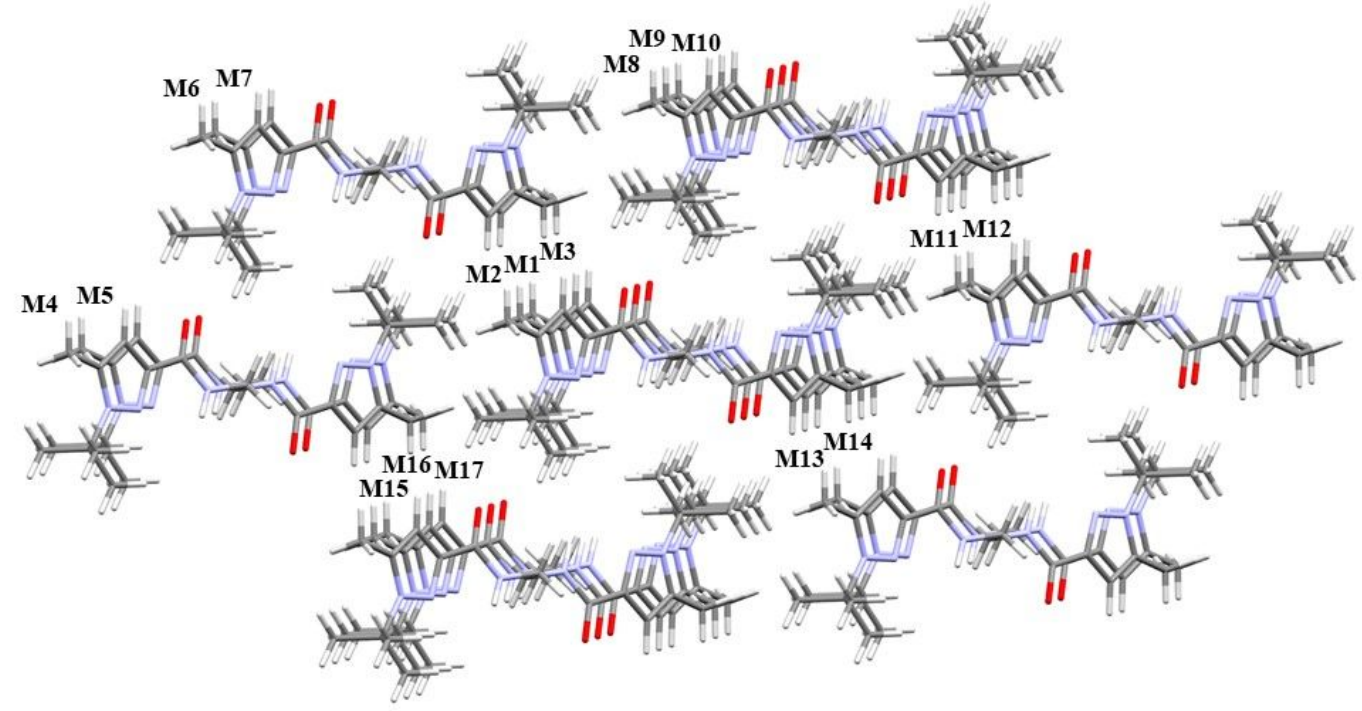

1aII 


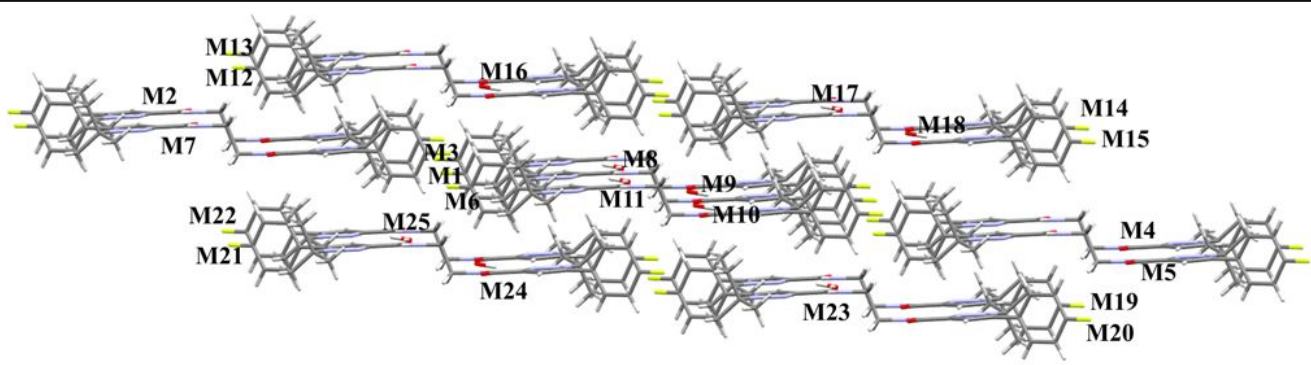

\section{1b}

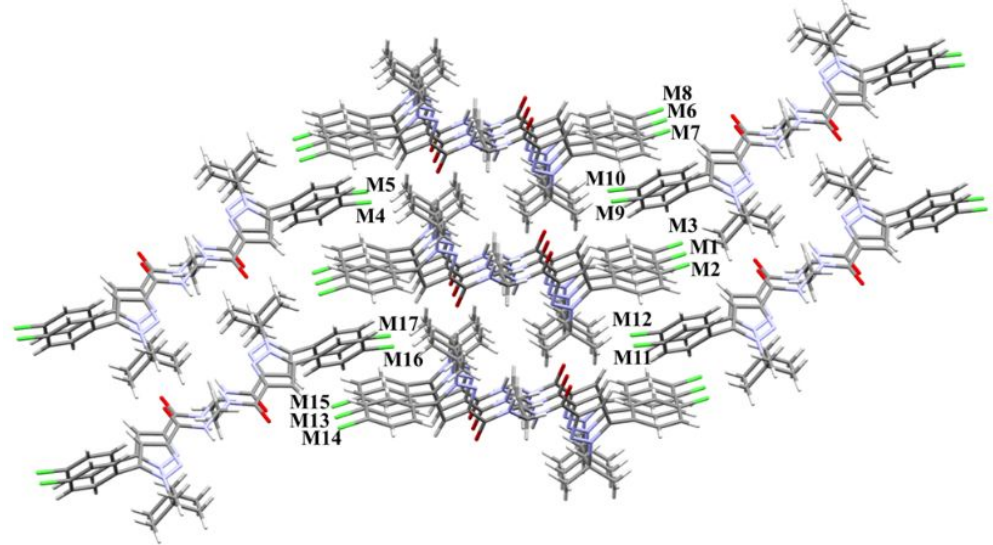

1c

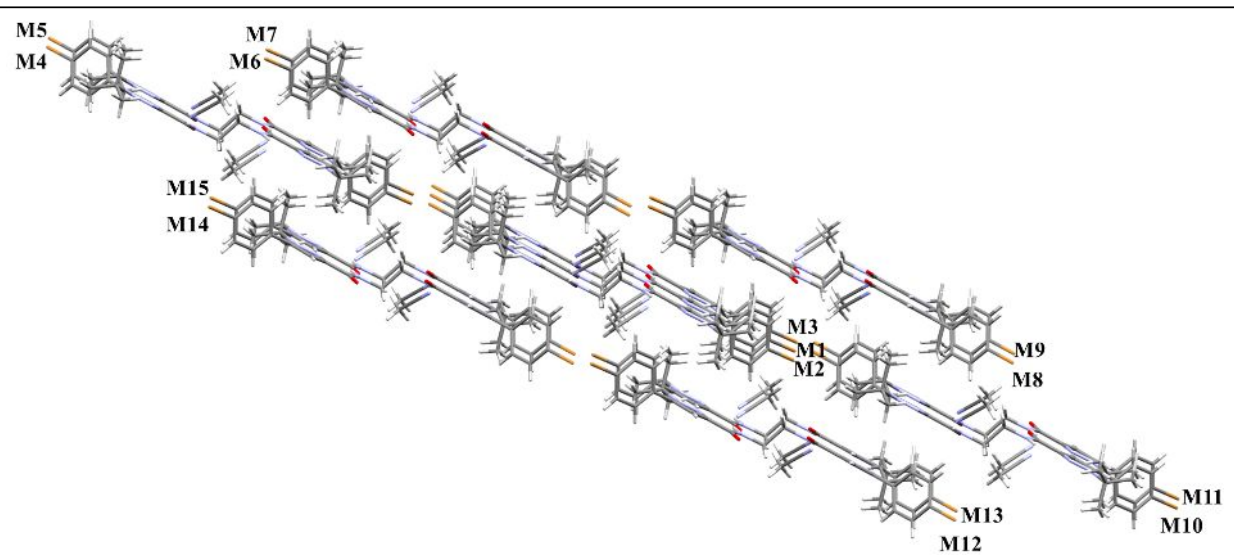

1d 


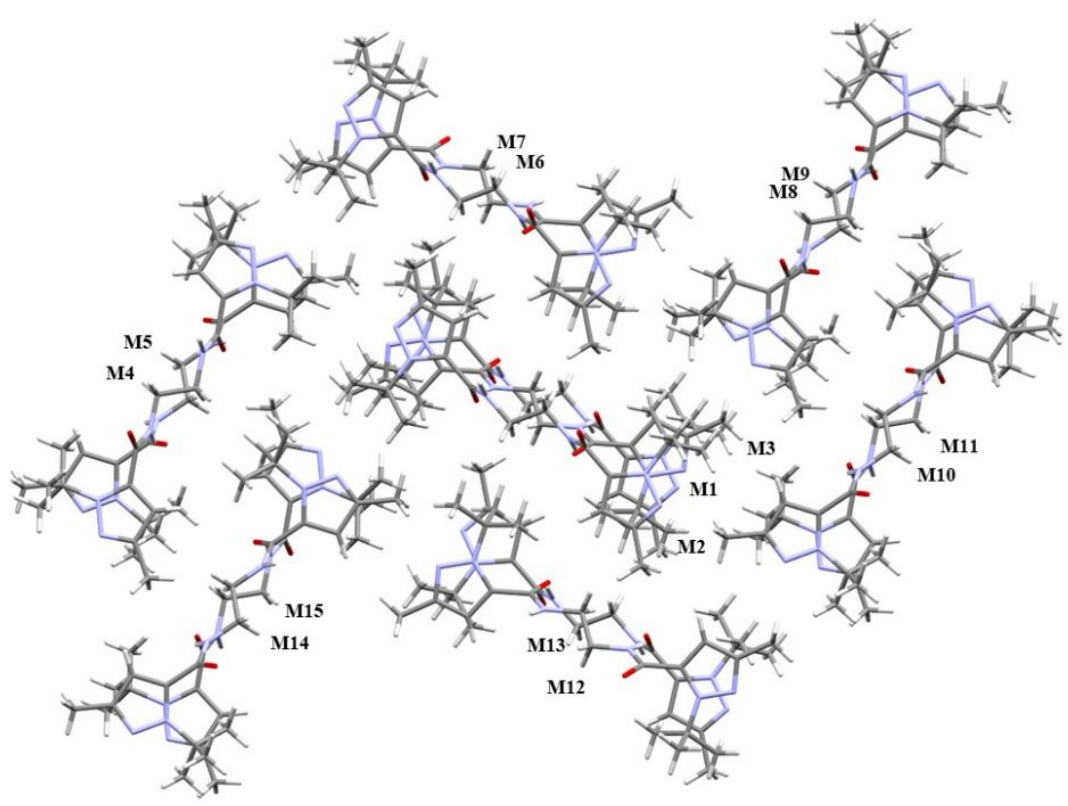

2aI

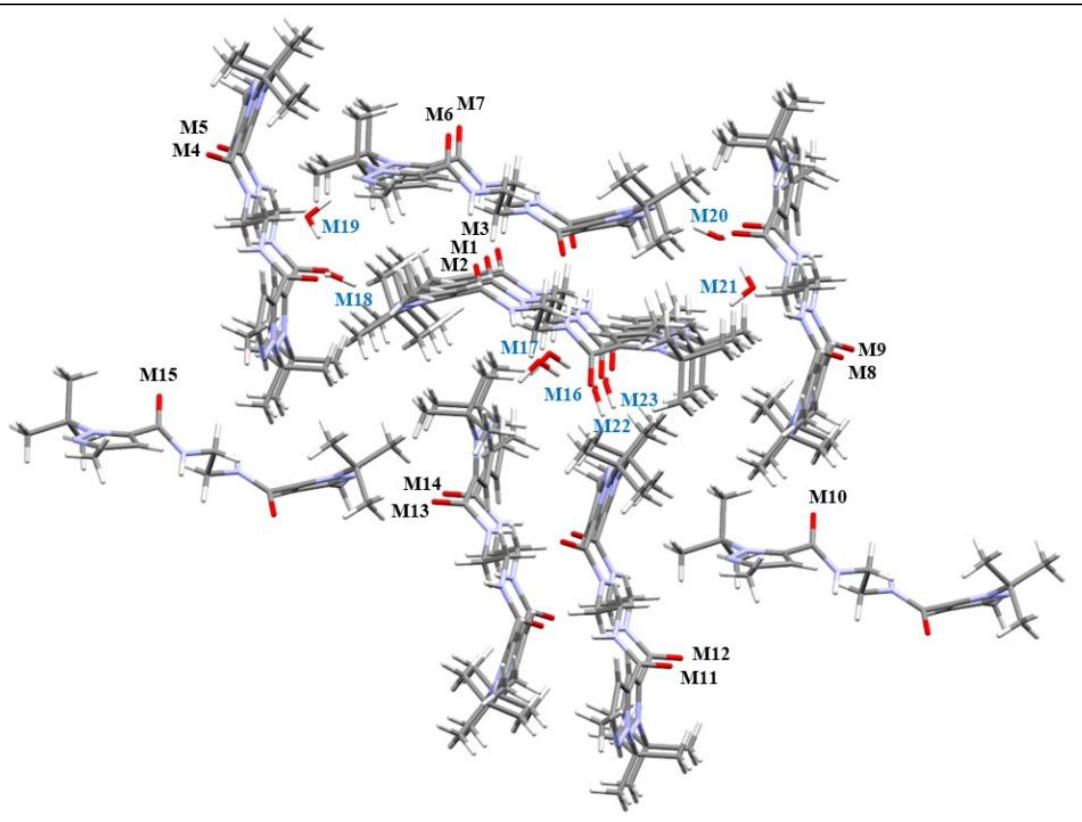

2aII 


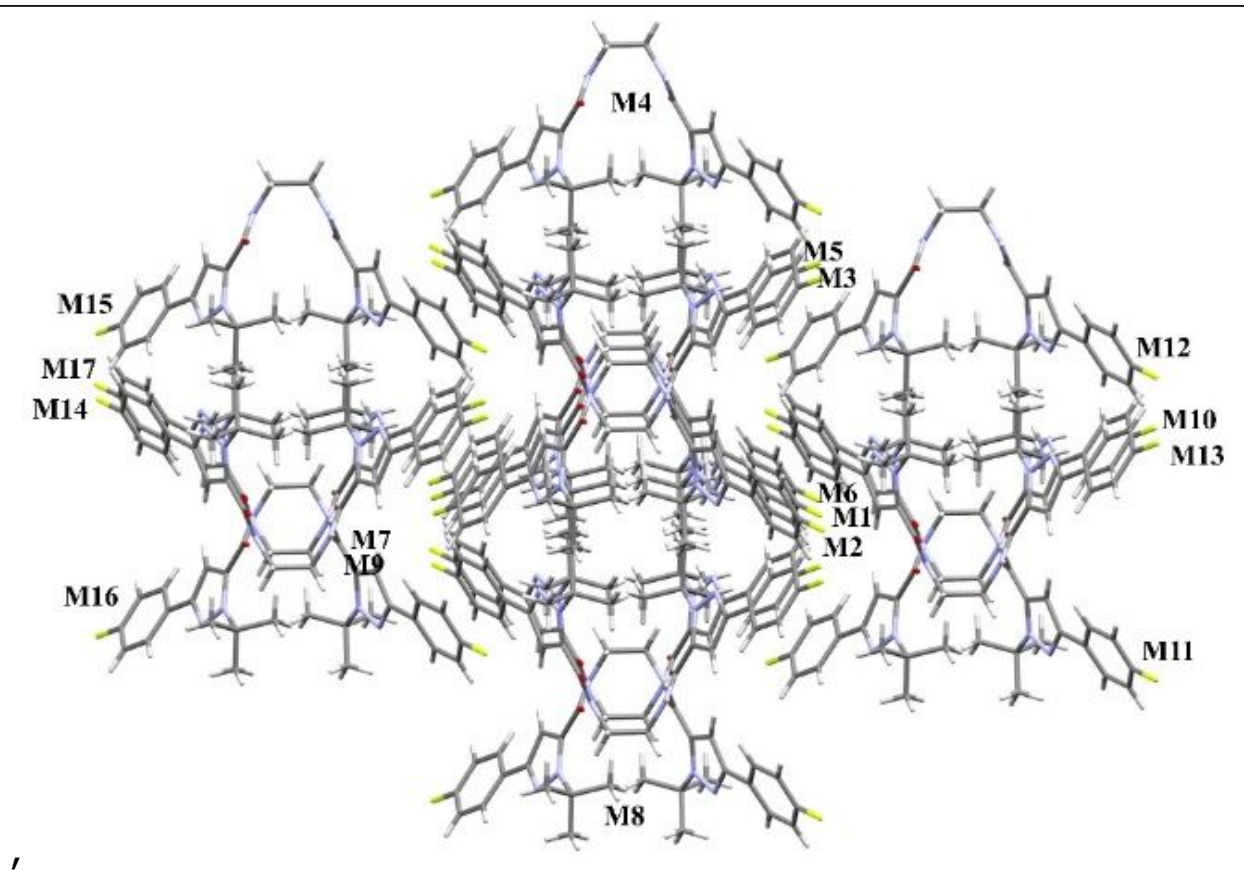

2b

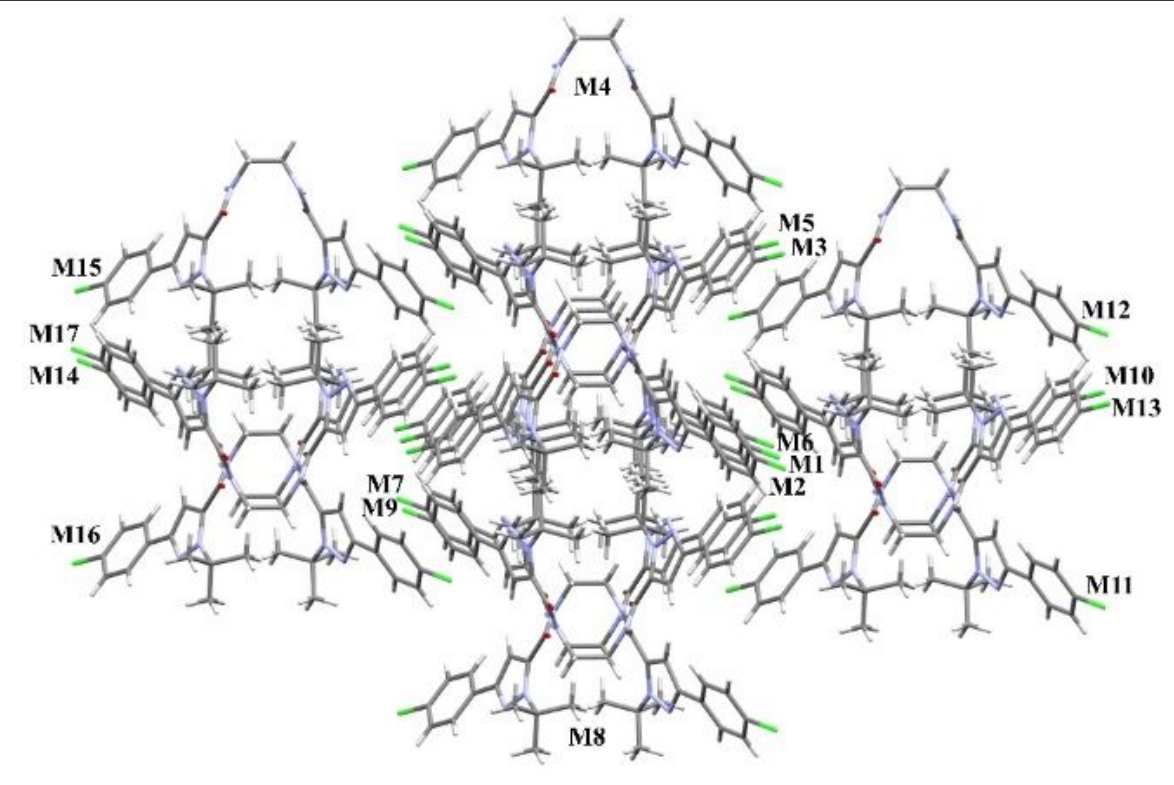

2c 


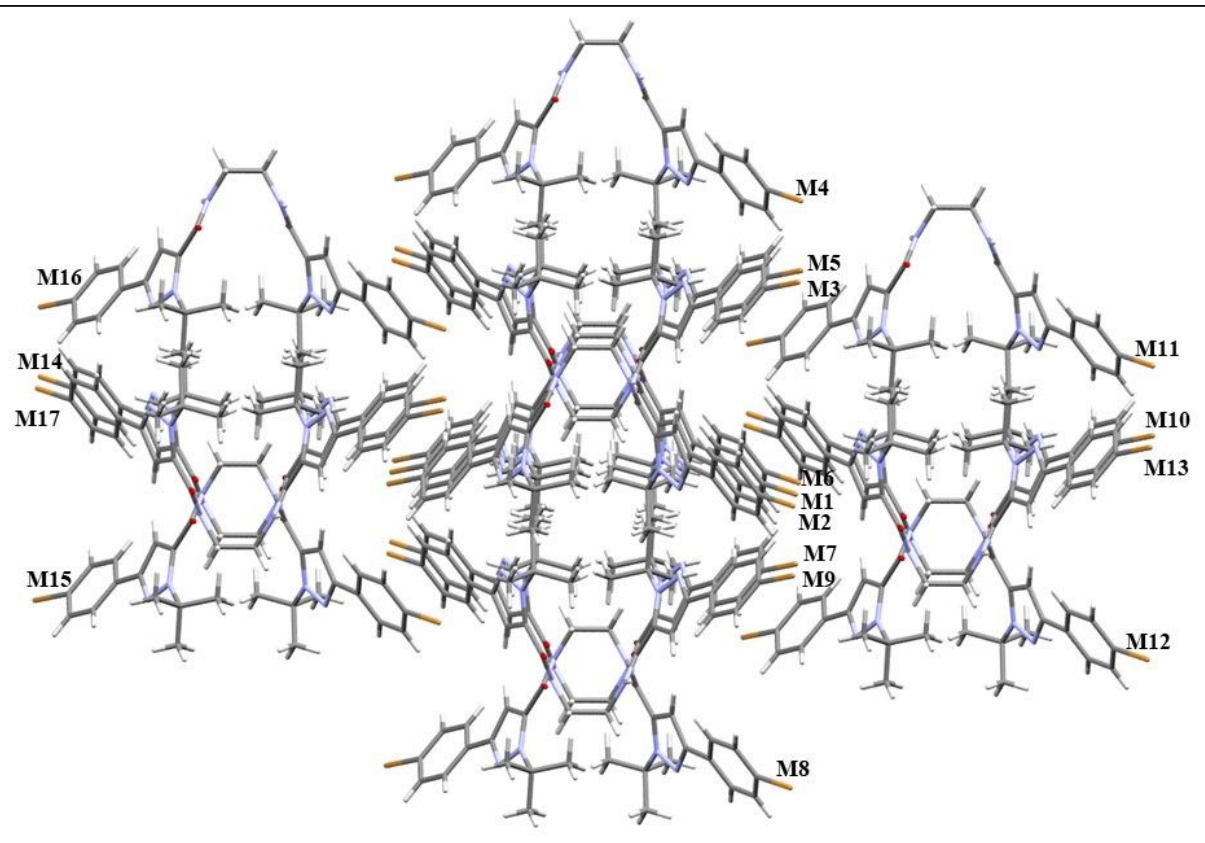

2b

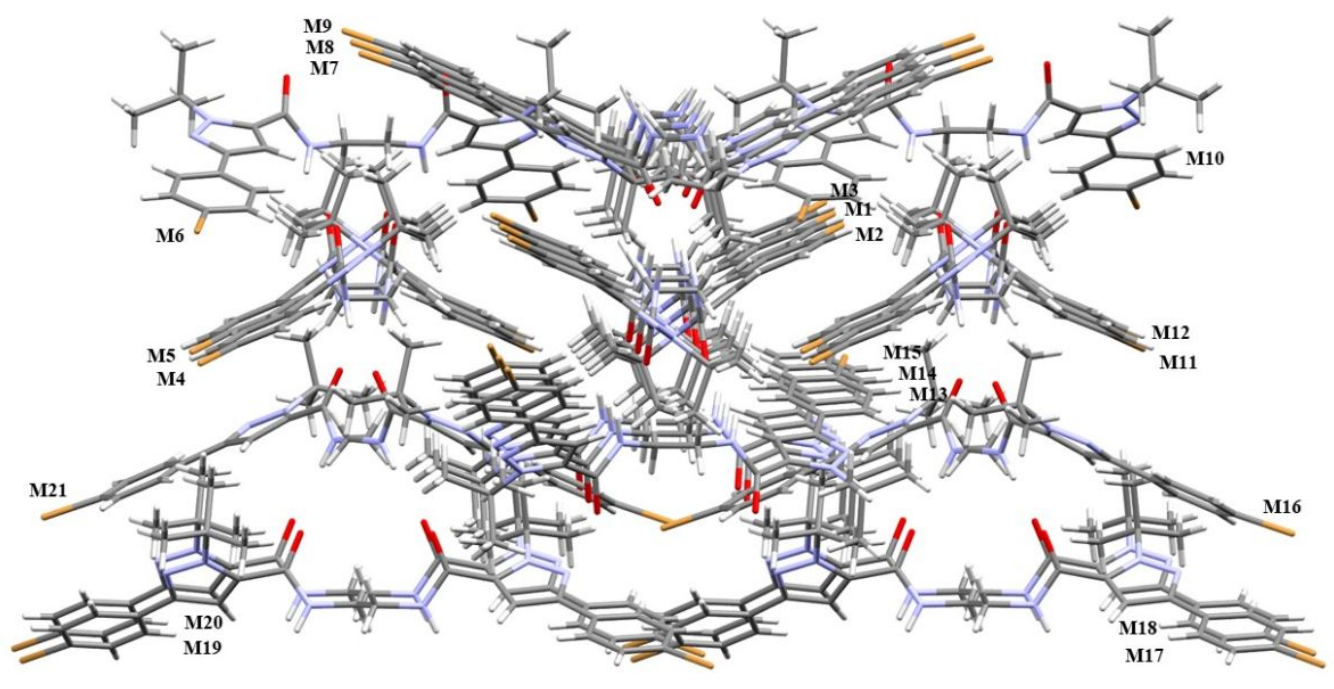

2dII 


\subsection{Topological and energetic data of compounds 1-2}

Table S7. Topological and energetic data of each dimer from the supramolecular cluster of compound 1aI.

\begin{tabular}{|c|c|c|c|c|c|}
\hline Dimer & Symmetry code ${ }^{a}$ & $\mathrm{C}_{M 1 \cdots M N}{ }^{b}$ & $\mathrm{G}_{\mathrm{M} 1 \cdots \mathrm{MN}}{ }^{\mathrm{c}}$ & $\mathrm{NC}_{\mathrm{M} 1 \cdots \mathrm{MN}}$ & $N G_{M 1 \cdots M N}$ \\
\hline $\mathrm{M} 1 \cdots \mathrm{M} 2$ & $1+x, y, z$ & 89.12 & -15.92 & 3.07 & 2.93 \\
\hline$M 1 \cdots M 3$ & $-1+x, y, z$ & 89.12 & -15.92 & 3.07 & 2.93 \\
\hline$M 1 \cdots M 4$ & $1-x, 1 / 2+y, 1.5-z$ & 35.67 & -7.18 & 1.23 & 1.32 \\
\hline 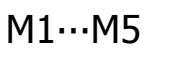 & $2-x, 1 / 2+y, 1.5-z$ & 16.94 & -3.18 & 0.58 & 0.59 \\
\hline 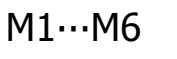 & $-x, 1 / 2+y, 1.5-z$ & 17.80 & -2.11 & 0.61 & 0.39 \\
\hline 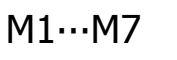 & $-1+x, 1+y, z$ & 20.54 & -6.49 & 0.71 & 1.20 \\
\hline$M 1 \cdots M 8$ & $-2+x, 1+y, z$ & 11.17 & -1.51 & 0.38 & 0.28 \\
\hline 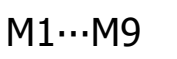 & $1+x, 1.5-y,-1 / 2+z$ & 35.67 & -7.18 & 1.23 & 1.32 \\
\hline 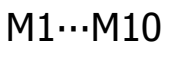 & $2+x, 1.5-y,-1 / 2+z$ & 17.80 & -2.11 & 0.61 & 0.39 \\
\hline 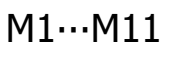 & $x, 1.5-y,-1 / 2+z$ & 16.94 & -3.18 & 0.58 & 0.59 \\
\hline 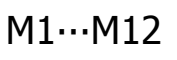 & $1+x, 1 / 2-y,-1 / 2+z$ & 35.67 & -7.18 & 1.23 & 1.32 \\
\hline M1 ‥M13 & $2+x, 1 / 2-y,-1 / 2+z$ & 17.80 & -2.11 & 0.61 & 0.39 \\
\hline 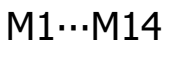 & $x, 1 / 2-y,-1 / 2+z$ & 16.94 & -3.18 & 0.58 & 0.59 \\
\hline 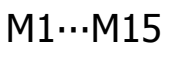 & $2+x,-1+y, z$ & 11.17 & -1.51 & 0.38 & 0.28 \\
\hline 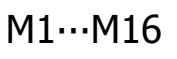 & $1+x,-1+y, z$ & 20.54 & -6.49 & 0.71 & 1.20 \\
\hline 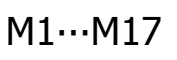 & $-1+x, 1 / 2-y, 1 / 2+z$ & 35.67 & -7.18 & 1.23 & 1.32 \\
\hline 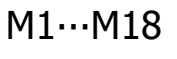 & $x, 1 / 2-y, 1 / 2+z$ & 16.94 & -3.18 & 0.58 & 0.59 \\
\hline 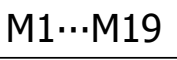 & $-2+x, 1 / 2-y, 1 / 2+z$ & 17.80 & -2.11 & 0.61 & 0.39 \\
\hline Total & & 523.30 & -97.71 & 18 & 18 \\
\hline
\end{tabular}

a Symmetry code obtained by the Mercury software. ${ }^{b} \AA^{2}$, contact surface obtained by ToposPro. ${ }^{\mathrm{c}} \mathrm{kcal} \mathrm{mol}^{-1}$, interaction energy obtained using $\omega \mathrm{B} 97 \mathrm{X}-\mathrm{D} / \mathrm{cc}-\mathrm{pVDZ}$ theory level.

Table S8. Topological and energetic data of each dimer from the supramolecular cluster of compound 1aII.

\begin{tabular}{|c|c|c|c|c|c|}
\hline Dimer & Symmetry code $^{a}$ & $\mathrm{C}_{\mathrm{M1} \cdots \mathrm{MN}}{ }^{\mathrm{b}}$ & $\mathrm{G}_{\mathrm{M} 1 \cdots \mathrm{MN}}{ }^{\mathrm{c}}$ & $\mathrm{NC}_{\mathrm{M} 1 \cdots \mathrm{MN}}$ & $N G_{M 1 \cdots M N}$ \\
\hline$M 1 \cdots M 2$ & $1+x, y, z$ & 88.53 & -16.77 & 2.70 & 2.74 \\
\hline 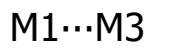 & $-1+x, y, z$ & 88.53 & -16.77 & 2.70 & 2.74 \\
\hline$M 1 \cdots M 4$ & $-1+x, 1+y,-1+z$ & 21.48 & -2.40 & 0.65 & 0.39 \\
\hline 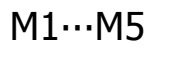 & $-2+x, 1+y,-1+z$ & 28.16 & -4.87 & 0.86 & 0.80 \\
\hline$M 1 \cdots M 6$ & $-1+x, y,-1+z$ & 7.78 & -1.11 & 0.69 & 0.18 \\
\hline$M 1 \cdots M 7$ & $-2+x, y,-1+z$ & 22.55 & -6.35 & 0.24 & 1.04 \\
\hline$M 1 \cdots M 8$ & $2+x,-1+y, z$ & 29,76 & -4.50 & 0.34 & 0.74 \\
\hline$M 1 \cdots M 9$ & $1+x,-1+y, z$ & 52.98 & -10.56 & 1.62 & 1.73 \\
\hline 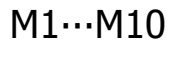 & $x,-1+y, z$ & 11,14 & -2.33 & 0.91 & 0.38 \\
\hline 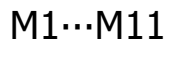 & $2+x,-1+y, 1+z$ & 28.16 & -4.87 & 0.86 & 0.80 \\
\hline 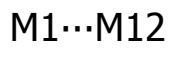 & $1+x,-1+y, 1+z$ & 21.48 & -2.58 & 0.65 & 0.42 \\
\hline 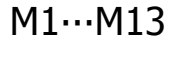 & $2+x, y, 1+z$ & 22.55 & -6.41 & 0.24 & 1.05 \\
\hline$M 1 \cdots M 14$ & $1+x, y, 1+z$ & 7.78 & -1.17 & 0.69 & 0.19 \\
\hline
\end{tabular}




\begin{tabular}{|c|c|c|c|c|c|}
\hline 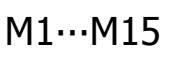 & $x, 1+y, z$ & 11.14 & -2.33 & 0.91 & 0.38 \\
\hline 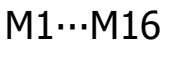 & $-1+x, 1+y, z$ & 52.98 & -10.33 & 1.62 & 1.69 \\
\hline M1…M17 & $-2+x, 1+y, z$ & 29.76 & -4.50 & 0.34 & 0.74 \\
\hline Total & & 524.76 & -97.84 & 16 & 16 \\
\hline
\end{tabular}


Table S9. Topological and energetic data of each dimer from the supramolecular cluster of compound 1 $\mathbf{b}$.

\begin{tabular}{|c|c|c|c|c|c|}
\hline Dimer & Symmetry code ${ }^{a}$ & $\mathrm{C}_{M 1 \cdots M N}{ }^{b}$ & $\mathrm{G}_{\mathrm{M} 1 \cdots \mathrm{MN}}{ }^{\mathrm{c}}$ & $\mathrm{NC}_{\mathrm{M1} \cdots \mathrm{MN}}$ & $N G_{M 1 \cdots M N}$ \\
\hline$M 1 \cdots M 2$ & $1+x, 1+y, 2+z$ & 42.20 & -4.84 & 1.49 & 0.95 \\
\hline M1‥M3 & $-1+x, y, z$ & 64.46 & -8.52 & 2.27 & 1.67 \\
\hline 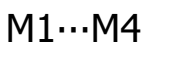 & $-2+x,-1+y,-2+z$ & 2.15 & -0.45 & 0.08 & 0.09 \\
\hline M1 $\cdots$ M5 & $-1+x,-1+y,-2+z$ & 42.20 & -5.08 & 1.49 & 1.00 \\
\hline 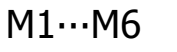 & $1+x, y, z$ & 64.46 & -8.52 & 2.27 & 1.67 \\
\hline 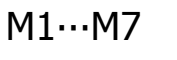 & $2+x, 1+y, 2+z$ & 2.15 & -0.01 & 0.08 & 0.00 \\
\hline$M 1 \cdots M 8$ & $x, y, 1+z$ & 18.30 & -1.11 & 0.65 & 0.22 \\
\hline 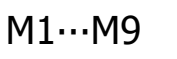 & $-x, 1-y, 1-z$ & 10.37 & -5.36 & 0.37 & 1.05 \\
\hline 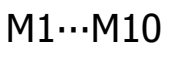 & $1-x, 1-y, 1-z$ & 18.30 & -1.11 & 0.65 & 0.22 \\
\hline 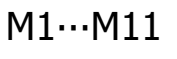 & $1+x, y, 1+z$ & 10.37 & -5.36 & 0.37 & 1.05 \\
\hline M1 ‥M12 & $1+x, 1+y, 1+z$ & 40.05 & -9.95 & 1.41 & 1.96 \\
\hline 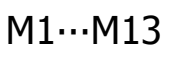 & $x, 1+y, 1+z$ & 39.31 & -7.44 & 1.39 & 1.46 \\
\hline 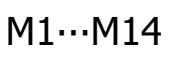 & $-1+x, y,-1+z$ & 35.66 & -9.71 & 1.26 & 1.91 \\
\hline 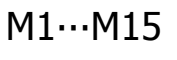 & $x, y,-1+z$ & 63.19 & -12.02 & 2.23 & 2.36 \\
\hline 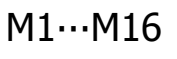 & $1-x, 2-y, 2-z$ & 9.18 & -0.47 & 0.32 & 0.09 \\
\hline 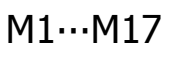 & $x, y, z$ & 10.23 & -0.67 & 0.36 & 0.13 \\
\hline 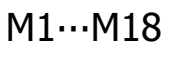 & $-x, 1-y,-z$ & 5.16 & -0.62 & 0.18 & 0.12 \\
\hline M1 ‥M19 & $-1+x,-1+y,-1+z$ & 40.05 & -9.95 & 1.41 & 1.96 \\
\hline M1 ‥M20 & $x,-1+y,-1+z$ & 39.31 & -7.44 & 1.39 & 1.46 \\
\hline$M 1 \cdots M 21$ & $1+x, y, 1+z$ & 35.66 & -9.71 & 1.26 & 1.91 \\
\hline$M 1 \cdots M 22$ & $x, y, 1+z$ & 63.19 & -12.02 & 2.23 & 2.36 \\
\hline M1 ‥M23 & $x,-1+y, z$ & 9.18 & -0.47 & 0.32 & 0.09 \\
\hline 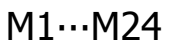 & $1-x, 1-y, 2-z$ & 10.23 & -0.67 & 0.36 & 0.13 \\
\hline 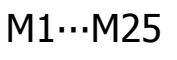 & $1+x, y, 2+z$ & 5.16 & -0.62 & 0.18 & 0.12 \\
\hline Total & & 680.52 & -122.09 & 24 & 24 \\
\hline
\end{tabular}

a Symmetry code obtained by the Mercury software. b $\AA^{2}$, contact surface obtained by ToposPro. ${ }^{\mathrm{c}} \mathrm{kcal} \mathrm{mol}^{-1}$, interaction energy obtained using $\omega \mathrm{B} 97 \mathrm{X}-\mathrm{D} / \mathrm{cc}-\mathrm{pVDZ}$ theory level. 
Table S10. Topological and energetic data of each dimer from the supramolecular cluster of compound 1c.

\begin{tabular}{|c|c|c|c|c|c|}
\hline Dimer & Symmetry code ${ }^{a}$ & $\mathrm{C}_{M 1 \cdots M N}{ }^{b}$ & $\mathrm{G}_{\mathrm{M} 1 \cdots M N}{ }^{\mathrm{c}}$ & $\mathrm{NC}_{\mathrm{M} 1 \cdots \mathrm{MN}}$ & $N G_{M 1 \cdots M N}$ \\
\hline$M 1 \cdots M 2$ & $-1+x, y, z$ & 128.07 & -23.17 & 2.98 & 3.01 \\
\hline M1..MM3 & $1+x, y, z$ & 128.07 & -23.17 & 2.98 & 3.01 \\
\hline 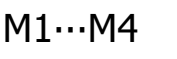 & $1.5+x, 1 / 2-y,-1 / 2+z$ & 23.29 & -3.61 & 0.54 & 0.47 \\
\hline 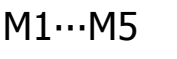 & $2.5+x, 1 / 2-y,-1 / 2+z$ & 34.10 & -5.46 & 0.79 & 0.71 \\
\hline M1..M6 & $1+x, 1+y, z$ & 57.58 & -11.79 & 1.34 & 1.53 \\
\hline 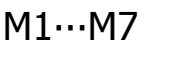 & $x, 1+y, z$ & 8.22 & -1.62 & 0.19 & 0.21 \\
\hline M1 $\cdots M 8$ & $2+x, 1+y, z$ & 34.75 & -6.92 & 0.81 & 0.90 \\
\hline 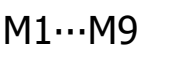 & $-2.5+x, 1 / 2-y, 1 / 2+z$ & 34.10 & -5.46 & 0.79 & 0.71 \\
\hline 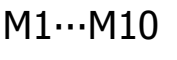 & $-1.5+x, 1 / 2-y, 1 / 2+z$ & 23.29 & -3.61 & 0.54 & 0.47 \\
\hline 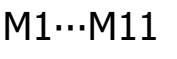 & $-2.5+x,-1 / 2-y, 1 / 2+z$ & 34.10 & -5.46 & 0.79 & 0.71 \\
\hline M1 $\cdots M 12$ & $-1.5+x,-1 / 2-y, 1 / 2+z$ & 23.29 & -3.61 & 0.54 & 0.47 \\
\hline 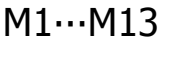 & $-1+x,-1+y, z$ & 57.58 & -11.79 & 1.34 & 1.53 \\
\hline 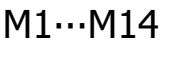 & $-2+x,-1+y, z$ & 34.75 & -6.92 & 0.81 & 0.90 \\
\hline 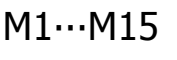 & $x,-1+y, z$ & 8.22 & -1.62 & 0.19 & 0.21 \\
\hline 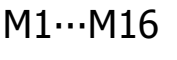 & $1.5+x,-1 / 2-y,-1 / 2+z$ & 23.29 & -3.61 & 0.54 & 0.47 \\
\hline M1 $\cdots M 17$ & $2.5+x,-1 / 2-y,-1 / 2+z$ & 34.10 & -5.46 & 0.79 & 0.71 \\
\hline Total & & 686.80 & -123.29 & 16 & 16 \\
\hline
\end{tabular}

a Symmetry code obtained by the Mercury software. ${ }^{b} \AA^{2}$, contact surface obtained by ToposPro. ${ }^{ } \mathrm{kcal} \mathrm{mol}{ }^{-1}$, interaction energy obtained using $\omega \mathrm{B} 97 \mathrm{X}-\mathrm{D} / \mathrm{cc}-\mathrm{pVDZ}$ theory level.

Table S11. Topological and energetic data of each dimer from the supramolecular cluster of compound 1d.

\begin{tabular}{|c|c|c|c|c|c|}
\hline Dimer & Symmetry code ${ }^{a}$ & $\mathrm{C}_{\mathrm{M1} \cdots \mathrm{MN}}{ }^{\mathrm{b}}$ & $\mathrm{G}_{\mathrm{M} 1 \cdots \mathrm{MN}}{ }^{\mathrm{c}}$ & $\mathrm{NC}_{\mathrm{M} 1 \cdots \mathrm{MN}}$ & $\mathrm{NG}_{\mathrm{M} 1 \cdots \mathrm{MN}}$ \\
\hline $\mathrm{M} 1 \cdots \mathrm{M} 2$ & $x,-1+y, z$ & 95.35 & -23.42 & 1.65 & 1.99 \\
\hline 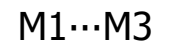 & $x, 1+y, z$ & 95.35 & -23.42 & 1.65 & 1.99 \\
\hline 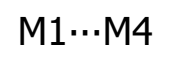 & $-1 / 2+x,-1 / 2+y,-1+z$ & 10.29 & -1.85 & 0.18 & 0.16 \\
\hline 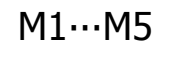 & $-1 / 2+x, 1 / 2+y,-1+z$ & 10.29 & -1.86 & 0.18 & 0.16 \\
\hline 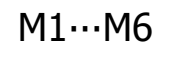 & $1 / 2-x, 1 / 2-y, 1-z$ & 59.79 & -6.39 & 1.03 & 0.54 \\
\hline 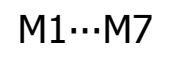 & $1 / 2-x, 1.5-y, 1-z$ & 87.39 & -20.95 & 1.51 & 1.78 \\
\hline 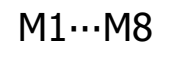 & $1-x, 1-y, 2-z$ & 105.31 & -19.09 & 1.82 & 1.62 \\
\hline 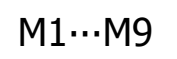 & $1-x, 2-y, 2-z$ & 31.68 & -8.88 & 0.55 & 0.75 \\
\hline M1 ‥M10 & $1 / 2+x,-1 / 2+y, 1+z$ & 10.29 & -1.84 & 0.18 & 0.16 \\
\hline M1 $\cdots M 11$ & $1 / 2+x, 1 / 2+y, 1+z$ & 10.29 & -1.84 & 0.18 & 0.16 \\
\hline 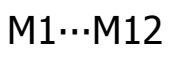 & $1.5-x, 1 / 2-y, 2-z$ & 59.79 & -6.37 & 1.03 & 0.54 \\
\hline M1 $\cdots$ M13 & $1.5-x, 1.5-y, 2-z$ & 98.24 & -20.92 & 1.70 & 1.78 \\
\hline 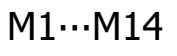 & $1-x, 1-y, 1-z$ & 105.31 & -19.09 & 1.82 & 1.62 \\
\hline M1 ‥M15 & $1-x, 2-y, 1-z$ & 31.68 & -8.90 & 0.55 & 0.76 \\
\hline Total & & 811.05 & -164.81 & 14 & 14 \\
\hline
\end{tabular}

a Symmetry code obtained by the Mercury software. ${ }^{b} \AA^{2}$, contact surface obtained by ToposPro. ${ }^{\mathrm{c}} \mathrm{kcal} \mathrm{mol}^{-1}$, interaction energy obtained using $\omega \mathrm{B} 97 \mathrm{X}-\mathrm{D} / \mathrm{cc}-\mathrm{pVDZ}$ theory level. 
Table S12. Topological and energetic data of each dimer from the supramolecular cluster of compound 2 aI.

\begin{tabular}{|c|c|c|c|c|c|}
\hline Dimer & Symmetry code ${ }^{a}$ & $\mathrm{C}_{M 1 \cdots M N}{ }^{b}$ & $\mathrm{G}_{\mathrm{M} 1 \cdots \mathrm{MN}}{ }^{\mathrm{c}}$ & $\mathrm{NC}_{\mathrm{M1} \cdots \mathrm{MN}}$ & $N G_{M 1 \cdots M N}$ \\
\hline$M 1 \cdots M 2$ & $x, y, z$ & 96.94 & -26.61 & 2.56 & 3.70 \\
\hline$M 1 \cdots M 3$ & $1+x, y, z$ & 96.94 & -26.61 & 2.56 & 3.70 \\
\hline 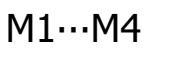 & $-x,-1 / 2+y,-1 / 2-z$ & 19.56 & -2.87 & 0.52 & 0.40 \\
\hline 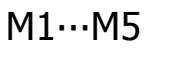 & $1-x,-1 / 2+y,-1 / 2-z$ & 19.90 & -3.12 & 0.52 & 0.43 \\
\hline 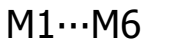 & $x, y,-1+z$ & 30.71 & -5.98 & 0.81 & 0.83 \\
\hline 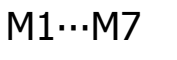 & $x, y,-1+z$ & 58.56 & -6.21 & 1.54 & 0.86 \\
\hline$M 1 \cdots M 8$ & $-x, 1 / 2+y,-1 / 2-z$ & 19.56 & -2.87 & 0.52 & 0.40 \\
\hline 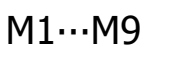 & $1-x, 1 / 2+y,-1 / 2-z$ & 20.29 & -2.76 & 0.53 & 0.38 \\
\hline 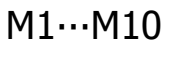 & $1-x, 1 / 2+y, 1 / 2-z$ & 19.90 & -2.87 & 0.52 & 0.40 \\
\hline 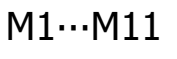 & $1-x, 1 / 2+y, 1 / 2-z$ & 19.56 & -2.87 & 0.52 & 0.40 \\
\hline M1 ‥M12 & $x, y, 1+z$ & 58.56 & -6.21 & 1.54 & 0.86 \\
\hline 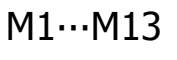 & $1+x, y, 1+z$ & 30.71 & -5.98 & 0.81 & 0.83 \\
\hline 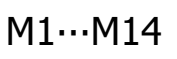 & $1-x,-1 / 2+y, 1 / 2-z$ & 20.29 & -2.76 & 0.53 & 0.38 \\
\hline 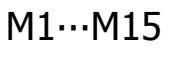 & $1-x,-1 / 2+y, 1 / 2-z$ & 19.56 & -2.87 & 0.52 & 0.40 \\
\hline Total & & 531.04 & -100.59 & 14 & 14 \\
\hline
\end{tabular}

a Symmetry code obtained by the Mercury software. b $\AA^{2}$, contact surface obtained by ToposPro. ${ }^{\mathrm{c}} \mathrm{kcal} \mathrm{mol}{ }^{-1}$, interaction energy obtained using $\omega \mathrm{B} 97 \mathrm{X}-\mathrm{D} / \mathrm{cc}-\mathrm{pVDZ}$ theory level. 
Table S13. Topological and energetic data of each dimer from the supramolecular cluster of compound 2aII.

\begin{tabular}{|c|c|c|c|c|c|}
\hline Dimer & Symmetry code a & $\mathrm{C}_{M 1 \cdots M N}{ }^{\mathrm{b}}$ & $\mathrm{G}_{\mathrm{M} 1 \cdots \mathrm{MN}}{ }^{\mathrm{c}}$ & $\mathrm{NC}_{\mathrm{M1} \cdots \mathrm{MN}}$ & $\mathrm{NG}_{\mathrm{M} 1 \cdots \mathrm{MN}}$ \\
\hline $\mathrm{M} 1 \cdots \mathrm{M} 2$ & $1+x, y, z$ & 32.43 & -3.10 & 1.44 & 0.69 \\
\hline M1‥M3 & $-1+x, y, z$ & 32.43 & -3.10 & 1.44 & 0.69 \\
\hline$M 1 \cdots M 4$ & $2-x, 1 / 2+y, 1.5-z$ & 7.51 & -1.48 & 0.33 & 0.33 \\
\hline 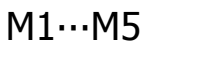 & $1-x, 1 / 2+y, 1.5-z$ & 32.60 & -5.26 & 1.45 & 1.18 \\
\hline 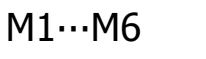 & $2-x, 1-y, 1-z$ & 42.19 & -6.19 & 1.87 & 1.39 \\
\hline M1 $\cdots M 7$ & $1-x, 1-y, 1-z$ & 92.40 & -23.92 & 4.11 & 5.36 \\
\hline$M 1 \cdots M 8$ & $x, 1 / 2-y,-1 / 2+z$ & 32.92 & -4.30 & 1.46 & 0.96 \\
\hline$M 1 \cdots M 9$ & $-1+x, 1 / 2-y,-1 / 2+z$ & 31.04 & -3.93 & 1.38 & 0.88 \\
\hline M1 $\cdots$ M10 & $1-x,-y, 1-z$ & 8.54 & -1.08 & 0.38 & 0.24 \\
\hline M1 $\cdots M 11$ & $2-x,-1 / 2+y, 1.5-z$ & 7.51 & -1.48 & 0.33 & 0.33 \\
\hline M1M12 & $1-x,-1 / 2+y, 1.5-z$ & 33.00 & -5.26 & 1.47 & 1.18 \\
\hline 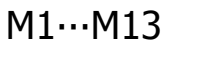 & $1+x, 1 / 2-y, 1 / 2+z$ & 23.52 & -3.93 & 1.04 & 0.88 \\
\hline 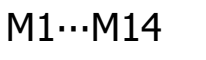 & $x, 1 / 2-y, 1 / 2+z$ & 32.92 & -4.30 & 1.46 & 0.96 \\
\hline 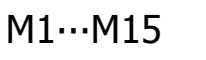 & $2-x, 1-y, 2-z$ & 6.61 & -1.49 & 0.29 & 0.33 \\
\hline 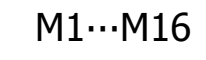 & $x, y, z$ & 13.81 & -5.12 & 0.61 & 1.15 \\
\hline 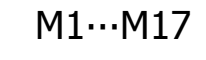 & $-1+x, y, z$ & 7.39 & -1.53 & 0.33 & 0.34 \\
\hline 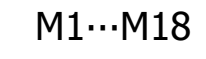 & $2-x, 1 / 2+y, 1.5-z$ & 11.23 & -5.04 & 0.50 & 1.13 \\
\hline M1 ‥M19 & $2-x, 1 / 2+y, 1.5-z$ & 5.57 & -1.49 & 0.25 & 0.33 \\
\hline$M 1 \cdots M 20$ & $x, 1 / 2-y,-1 / 2+z$ & 0.27 & -0.03 & 0.01 & 0.01 \\
\hline$M 1 \cdots M 21$ & $-1+x, 1 / 2-y,-1 / 2+z$ & 19.51 & -5.36 & 0.87 & 1.20 \\
\hline 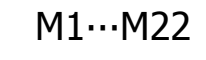 & $x, y, z$ & 17.45 & -9.58 & 0.78 & 2.15 \\
\hline M1 $\cdots M 23$ & $-1+x, y, z$ & 4.31 & -1.18 & 0.19 & 0.26 \\
\hline Total & & 495.16 & -98.15 & 22 & 22 \\
\hline
\end{tabular}

a Symmetry code obtained by the Mercury software. b $\AA^{2}$, contact surface obtained by ToposPro. ${ }^{\mathrm{c}} \mathrm{kcal} \mathrm{mol}^{-1}$, interaction energy obtained using $\omega \mathrm{B} 97 \mathrm{X}-\mathrm{D} / \mathrm{cc}-\mathrm{pVDZ}$ theory level. 
Table S14. Topological and energetic data of each dimer from the supramolecular cluster of compound $\mathbf{2 b}$.

\begin{tabular}{|c|c|c|c|c|c|}
\hline Dimer & Symmetry code ${ }^{a}$ & $\mathrm{C}_{\mathrm{M} 1 \cdots \mathrm{MN}}{ }^{\mathrm{b}}$ & $\mathrm{G}_{\mathrm{M} 1 \cdots \mathrm{MN}}{ }^{\mathrm{c}}$ & $\mathrm{NC}_{\mathrm{M} 1 \cdots \mathrm{MN}}$ & $\mathrm{NG}_{\mathrm{M} 1 \cdots \mathrm{MN}}$ \\
\hline$M 1 \cdots M 2$ & $x, y, 1+z$ & 20.53 & -3.75 & 0.53 & 0.55 \\
\hline M1‥M3 & $-x, 1-y, 1-z$ & 82.95 & -21.91 & 2.16 & 3.22 \\
\hline$M 1 \cdots M 4$ & $x, 1+y, z$ & 6.54 & -1.25 & 0.17 & 0.18 \\
\hline 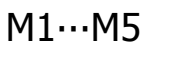 & $-x, 1-y,-z$ & 82.95 & -22.05 & 2.16 & 3.25 \\
\hline$M 1 \cdots M 6$ & $x, y,-1+z$ & 20.53 & -3.75 & 0.53 & 0.55 \\
\hline$M 1 \cdots M 7$ & $-x,-y,-z$ & 101.44 & -13.52 & 2.64 & 1.99 \\
\hline$M 1 \cdots M 8$ & $x,-1+y, z$ & 6.54 & -1.25 & 0.17 & 0.18 \\
\hline 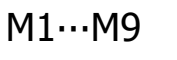 & $-x,-y, 1-z$ & 101.44 & -13.35 & 2.64 & 1.96 \\
\hline 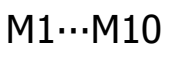 & $1 / 2-x, 1 / 2-y, 1-z$ & 57.66 & -9.07 & 1.50 & 1.33 \\
\hline 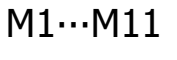 & $1 / 2+x,-1 / 2+y, 1+z$ & 14.84 & -1.61 & 0.39 & 0.24 \\
\hline 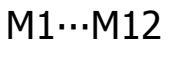 & $1 / 2+x, 1 / 2+y, 1+z$ & 14.84 & -1.61 & 0.39 & 0.24 \\
\hline 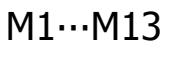 & $1 / 2-x, 1 / 2-y, 2-z$ & 8.87 & -1.65 & 0.23 & 0.24 \\
\hline 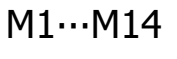 & $-1 / 2-x, 1 / 2-y,-z$ & 57.66 & -9.07 & 1.50 & 1.33 \\
\hline 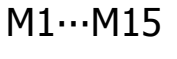 & $-1 / 2+x, 1 / 2+y,-1+z$ & 14.84 & -1.61 & 0.39 & 0.24 \\
\hline 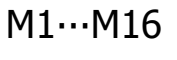 & $-1 / 2+x,-1 / 2+y,-1+z$ & 14.84 & -1.61 & 0.39 & 0.24 \\
\hline 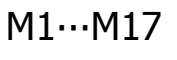 & $-1 / 2-x, 1 / 2-y,-1-z$ & 8.87 & -1.65 & 0.23 & 0.24 \\
\hline Total & & 615.34 & -108.73 & 16 & 16 \\
\hline
\end{tabular}

a Symmetry code obtained by the Mercury software. b $\AA^{2}$, contact surface obtained by ToposPro. ${ }^{ } \mathrm{kcal} \mathrm{mol}{ }^{-1}$, interaction energy obtained using $\omega \mathrm{B} 97 \mathrm{X}-\mathrm{D} / \mathrm{cc}-\mathrm{pVDZ}$ theory level. 
Table S15. Topological and energetic data of each dimer from the supramolecular cluster of compound 2c.

\begin{tabular}{|c|c|c|c|c|c|}
\hline Dimer & Symmetry code ${ }^{a}$ & $\mathrm{C}_{\mathrm{M} 1 \cdots \mathrm{MN}}{ }^{\mathrm{b}}$ & $\mathrm{G}_{\mathrm{M} 1 \cdots \mathrm{MN}}{ }^{\mathrm{c}}$ & $\mathrm{NC}_{\mathrm{M} 1 \cdots \mathrm{MN}}$ & $N G_{M 1 \cdots M N}$ \\
\hline $\mathrm{M} 1 \cdots \mathrm{M} 2$ & $x, y, 1+z$ & 23.59 & -4.19 & 0.59 & 0.59 \\
\hline$M 1 \cdots M 3$ & $-x, 2-y, 1-z$ & 87.14 & -23.21 & 2.17 & 3.27 \\
\hline M1...M4 & $x, 1+y, z$ & 8.22 & -1.32 & 0.21 & 0.19 \\
\hline 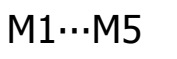 & $-x, 2-y,-z$ & 87.14 & -23.22 & 2.17 & 3.27 \\
\hline M1..M M6 & $x, y,-1+z$ & 23.59 & -4.19 & 0.59 & 0.59 \\
\hline M1 $\cdots M 7$ & $-x, 1-y,-z$ & 106.06 & -13.70 & 2.65 & 1.93 \\
\hline M1 $\cdots$ M8 & $x,-1+y, z$ & 8.22 & -1.32 & 0.21 & 0.19 \\
\hline 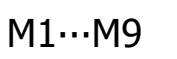 & $-x, 1-y, 1-z$ & 106.06 & -13.70 & 2.65 & 1.93 \\
\hline M1 $\cdots$ M10 & $1 / 2-x, 1.5-y, 1-z$ & 59.18 & -9.68 & 1.48 & 1.36 \\
\hline M1 $\cdots M 11$ & $1 / 2+x,-1 / 2+y, 1+z$ & 13.80 & -1.53 & 0.34 & 0.22 \\
\hline M1M12 & $1 / 2+x, 1 / 2+y, 1+z$ & 13.80 & -1.53 & 0.34 & 0.22 \\
\hline 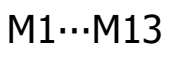 & $1 / 2-x, 1.5-y, 2-z$ & 8.55 & -1.60 & 0.21 & 0.23 \\
\hline 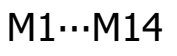 & $-1 / 2-x, 1.5-y,-z$ & 59.80 & -9.68 & 1.48 & 1.36 \\
\hline 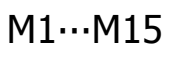 & $-1 / 2+x, 1 / 2+y,-1+z$ & 13.80 & -1.53 & 0.34 & 0.22 \\
\hline 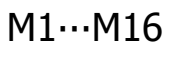 & $-1 / 2+x,-1 / 2+y,-1+z$ & 13.80 & -1.53 & 0.34 & 0.22 \\
\hline 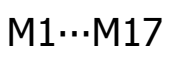 & $-1 / 2-x, 1.5-y,-1-z$ & 8.55 & -1.60 & 0.21 & 0.23 \\
\hline Total & & 641.30 & -113.52 & 16 & 16 \\
\hline
\end{tabular}

a Symmetry code obtained by the Mercury software. b $\AA^{2}$, contact surface obtained by ToposPro. ${ }^{\mathrm{c}} \mathrm{kcal} \mathrm{mol}{ }^{-1}$, interaction energy obtained using $\omega \mathrm{B} 97 \mathrm{X}-\mathrm{D} / \mathrm{cc}-\mathrm{pVDZ}$ theory level.

Table S16. Topological and energetic data of each dimer from the supramolecular cluster of compound $\mathbf{2 d I}$.

\begin{tabular}{|c|c|c|c|c|c|}
\hline Dimer & Symmetry code ${ }^{a}$ & $\mathrm{C}_{\mathrm{M} 1 \cdots \mathrm{MN}}{ }^{\mathrm{b}}$ & $\mathrm{G}_{\mathrm{M1} \cdots \mathrm{MN}}{ }^{\mathrm{c}}$ & $\mathrm{NC}_{\mathrm{M} 1 \cdots \mathrm{MN}}$ & $N G_{M 1 \cdots M N}$ \\
\hline $\mathrm{M} 1 \cdots \mathrm{M} 2$ & $x, y, 1+z$ & 23.64 & -4.32 & 0.58 & 0.60 \\
\hline$M 1 \cdots M 3$ & $-x, 2-y, 1-z$ & 88.49 & -22.86 & 2.17 & 3.15 \\
\hline M1..M4 & $x, 1+y, z$ & 9.86 & -1.51 & 0.24 & 0.21 \\
\hline 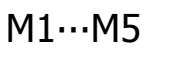 & $-x, 2-y,-z$ & 88.49 & -22.86 & 2.17 & 3.15 \\
\hline 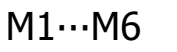 & $x, y,-1+z$ & 23.64 & -4.32 & 0.58 & 0.60 \\
\hline M1...M7 & $-x, 1-y,-z$ & 109.30 & -14.08 & 2.68 & 1.94 \\
\hline$M 1 \cdots M 8$ & $x,-1+y, z$ & 9.86 & -1.51 & 0.24 & 0.21 \\
\hline 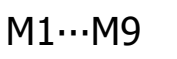 & $-x, 1-y, 1-z$ & 109.30 & -14.08 & 2.68 & 1.94 \\
\hline 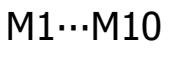 & $1 / 2-x, 1.5-y, 1-z$ & 58.30 & -9.99 & 1.43 & 1.38 \\
\hline M1 $\cdots M 11$ & $1 / 2+x, 1 / 2+y, 1+z$ & 13.69 & -1.85 & 0.34 & 0.26 \\
\hline 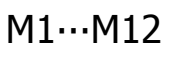 & $1 / 2+x,-1 / 2+y, 1+z$ & 13.69 & -1.85 & 0.34 & 0.26 \\
\hline M1...M13 & $1 / 2-x, 1.5-y, 2-z$ & 8.76 & -1.55 & 0.22 & 0.21 \\
\hline 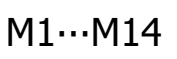 & $-1 / 2-x, 1.5-y,-1-z$ & 8.76 & -1.55 & 0.22 & 0.21 \\
\hline 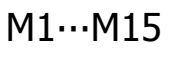 & $-1 / 2+x,-1 / 2+y,-1+z$ & 13.69 & -1.85 & 0.34 & 0.26 \\
\hline 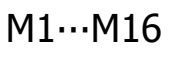 & $-1 / 2+x, 1 / 2+y,-1+z$ & 13.69 & -1.85 & 0.34 & 0.26 \\
\hline 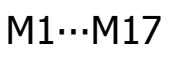 & $-1 / 2-x, 1.5-y,-z$ & 58.30 & -9.99 & 1.43 & 1.38 \\
\hline Total & & 651.46 & -116.02 & 16 & 16 \\
\hline
\end{tabular}

a Symmetry code obtained by the Mercury software. b $\AA^{2}$, contact surface obtained by ToposPro. ${ }^{\mathrm{c}} \mathrm{kcal} \mathrm{mol}^{-1}$, interaction energy obtained using $\omega \mathrm{B} 97 \mathrm{X}-\mathrm{D} / \mathrm{cc}-\mathrm{pVDZ}$ theory level. 
Table S17. Topological and energetic data of each dimer from the supramolecular cluster of compound 2dII.

\begin{tabular}{|c|c|c|c|c|c|}
\hline Dimer & Symmetry code $^{a}$ & $\mathrm{C}_{M 1 \cdots M N}{ }^{b}$ & $\mathrm{G}_{\mathrm{M} 1 \cdots M N}{ }^{\mathrm{c}}$ & $\mathrm{NC}_{\mathrm{M1} \cdots \mathrm{MN}}$ & $N G_{M 1 \cdots M N}$ \\
\hline 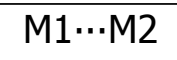 & $x,-1+y, z$ & 31.76 & -2.95 & 0.83 & 0.48 \\
\hline M1‥M3 & $x, 1+y, z$ & 31.76 & -2.85 & 0.83 & 0.48 \\
\hline 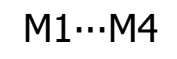 & $-x, 1-y, 1-z$ & 64.62 & -9.42 & 1.70 & 1.54 \\
\hline 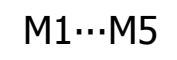 & $-x, 2-y, 1-z$ & 5.11 & -0.12 & 0.13 & 0.02 \\
\hline M1..M6 & $-x, 1-y, 1-z$ & 61.55 & -11.73 & 1.62 & 1.91 \\
\hline 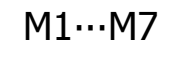 & $1-y,-1 / 2+x, 1 / 2+z$ & 20.81 & -2.50 & 0.55 & 0.41 \\
\hline M1...M8 & $1-y, 1 / 2+x, 1 / 2+z$ & 110.82 & -24.46 & 2.91 & 3.99 \\
\hline 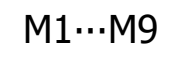 & $1-y, 1.5+x, 1 / 2+z$ & 20.81 & -2.50 & 0.55 & 0.41 \\
\hline 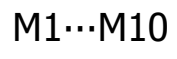 & $1-x, 2-y, 1-z$ & 61.55 & -11.73 & 1.62 & 1.91 \\
\hline$M 1 \cdots M 11$ & $1 / 2+x,-1 / 2+y, 1-z$ & 5.11 & -0.12 & 0.13 & 0.02 \\
\hline$M 1 \cdots M 12$ & $1-x, 2-y, 1-z$ & 64.62 & -9.42 & 1.70 & 1.54 \\
\hline$M 1 \cdots M 13$ & $x,-1+y, z$ & 13.72 & -1.04 & 0.36 & 0.17 \\
\hline$M 1 \cdots M 14$ & $x, y, z$ & 149.08 & -29.86 & 3.92 & 4.87 \\
\hline 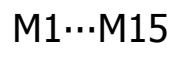 & $1 / 2-x, 2.5-y, z$ & 13.72 & -1.04 & 0.36 & 0.17 \\
\hline$M 1 \cdots M 16$ & $y, 1 / 2-x, 1 / 2-z$ & 44.12 & -4.69 & 1.16 & 0.76 \\
\hline$M 1 \cdots M 17$ & $y, 1 / 2-x, 1 / 2-z$ & 4.41 & -0.89 & 0.12 & 0.14 \\
\hline$M 1 \cdots M 18$ & $y, 1.5-x, 1 / 2-z$ & 4.41 & -0.89 & 0.12 & 0.14 \\
\hline 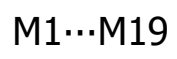 & $-1+y, 1 / 2-x, 1 / 2-z$ & 4.41 & -0.89 & 0.12 & 0.14 \\
\hline$M 1 \cdots M 20$ & $-1+y, 1.5-x, 1 / 2-z$ & 4.41 & -0.89 & 0.12 & 0.14 \\
\hline$M 1 \cdots M 21$ & $-1+y, 1.5-x, 1 / 2-z$ & 44.12 & -4.69 & 1.16 & 0.76 \\
\hline Cluster & & 760.92 & -122.66 & 20 & 20 \\
\hline
\end{tabular}

a Symmetry code obtained by the Mercury software. ${ }^{b} \AA^{2}$, contact surface obtained by ToposPro. ${ }^{\mathrm{c}} \mathrm{kcal} \mathrm{mol}^{-1}$, interaction energy obtained using $\omega \mathrm{B} 97 \mathrm{X}-\mathrm{D} / \mathrm{cc}-\mathrm{pVDZ}$ theory level. 


\subsection{Normalized Contact Area and Energy}

Table S18. Normalized contact area and energy of compounds 1-2.

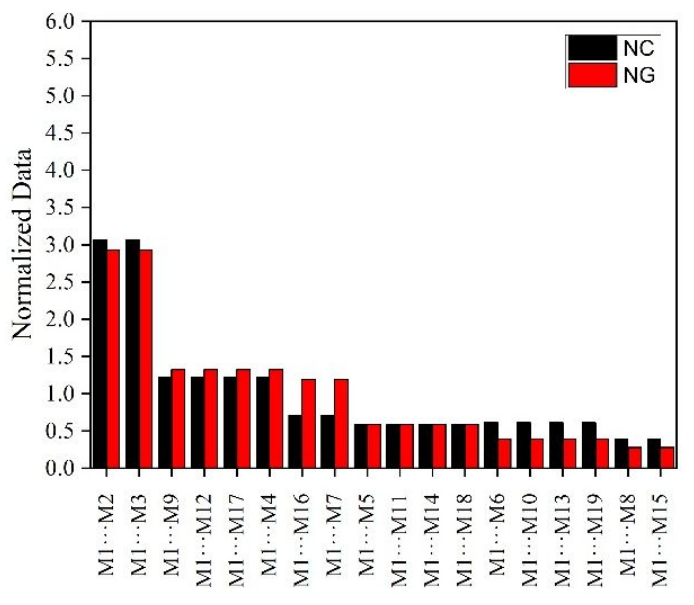

1aI

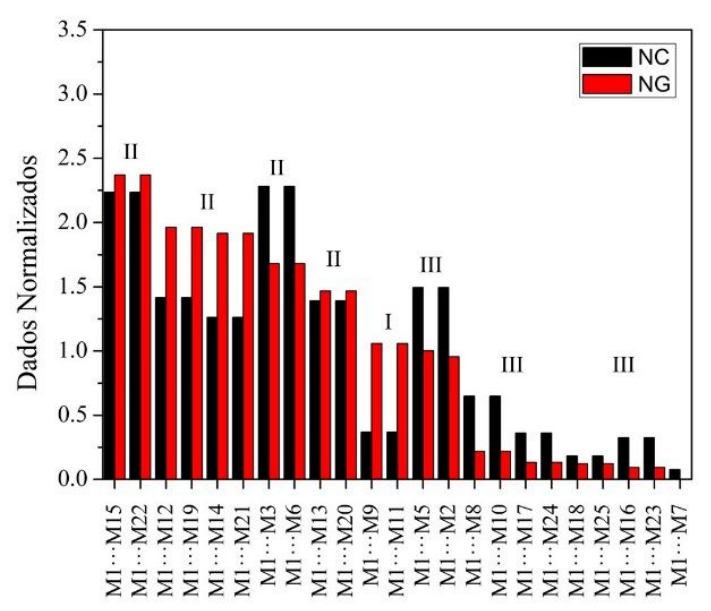

1b

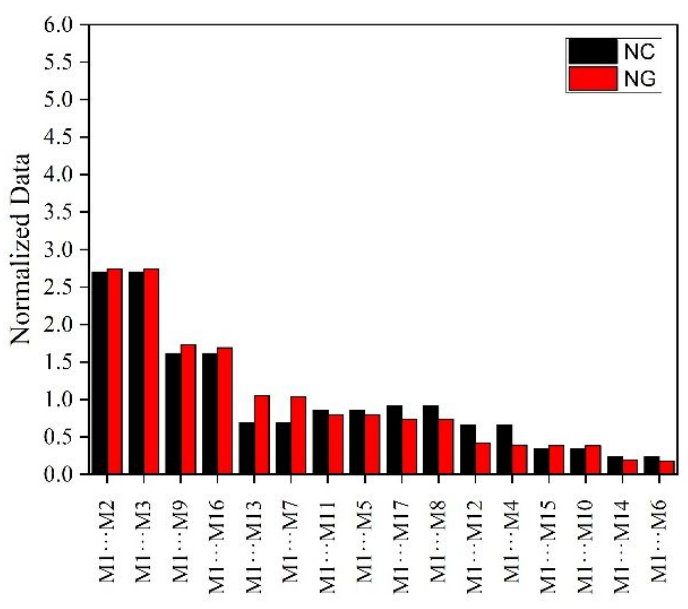

1aII

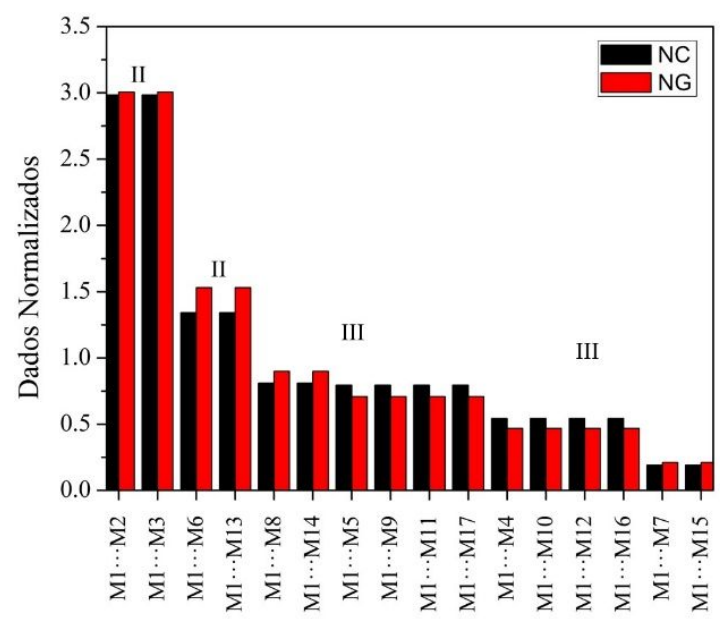

1c

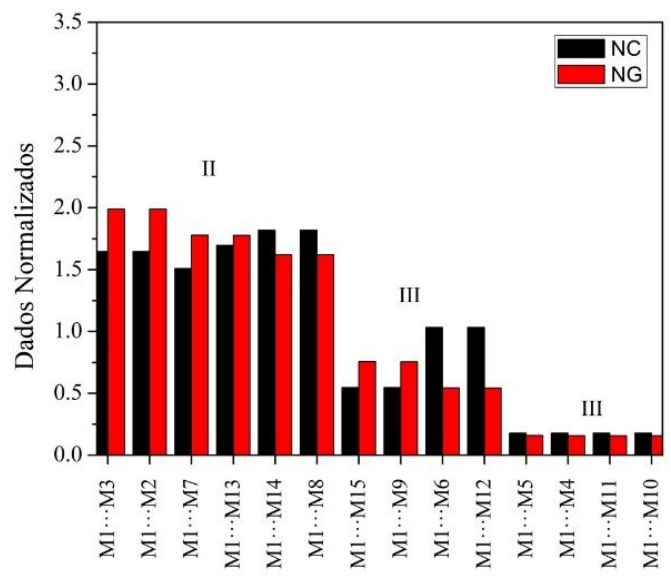

1d 


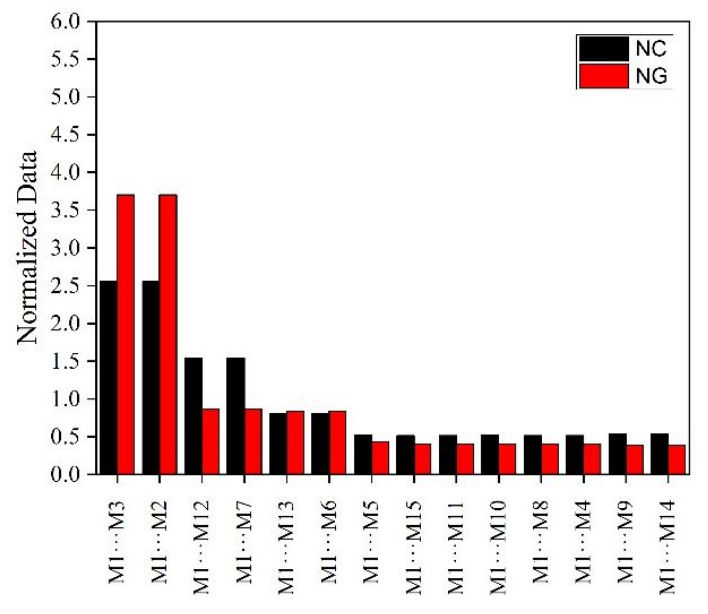

2aI

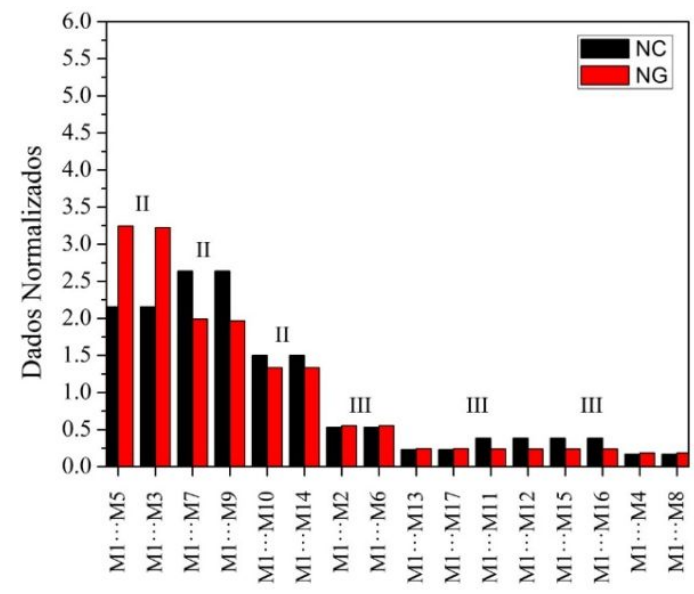

2b

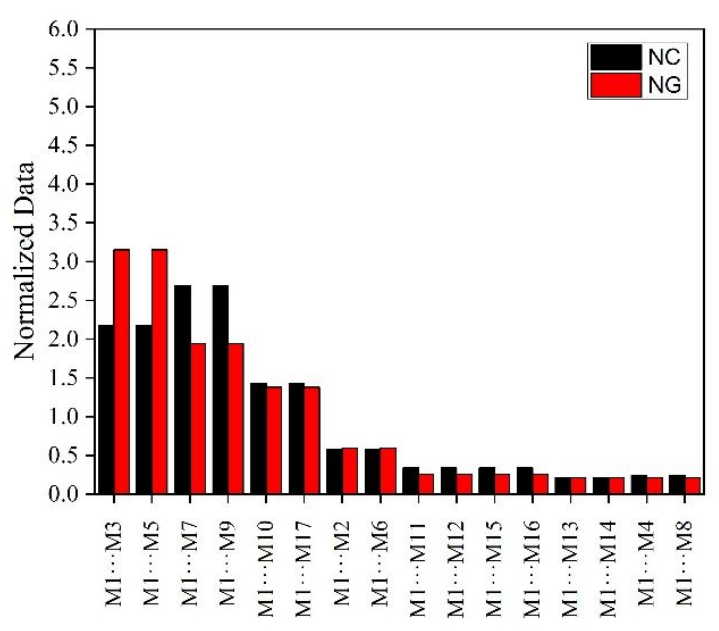

2dI

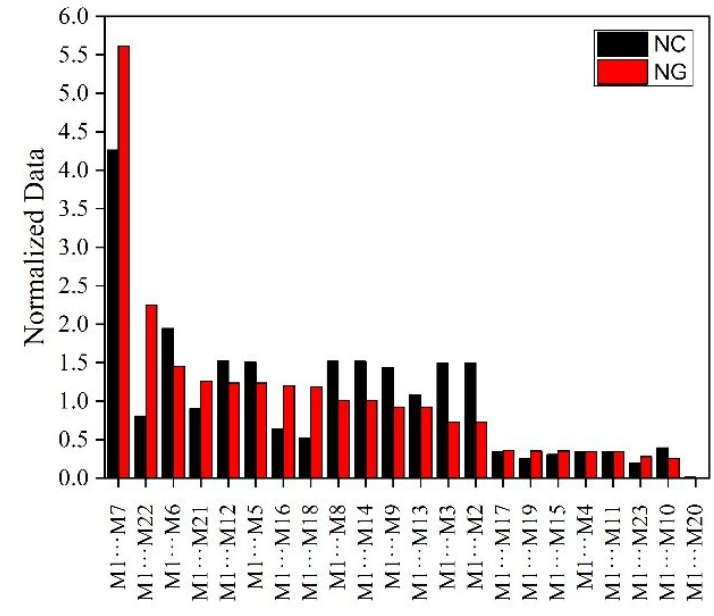

2aII

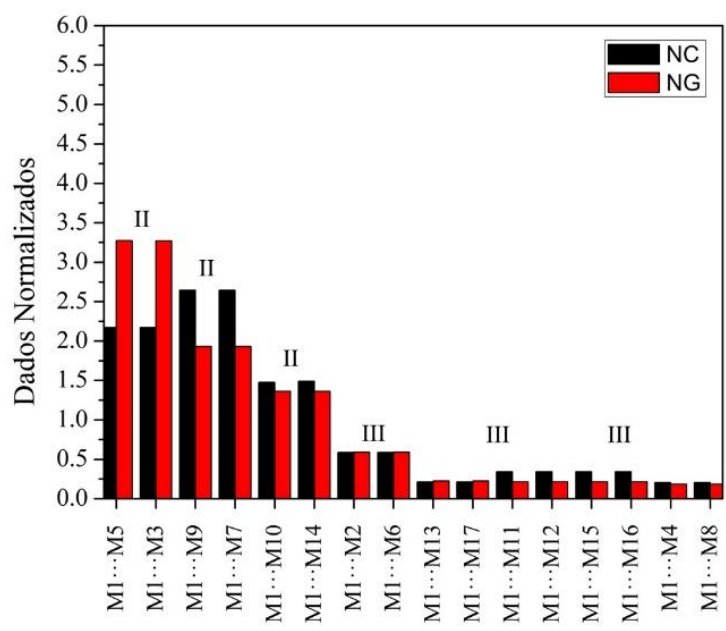

2c

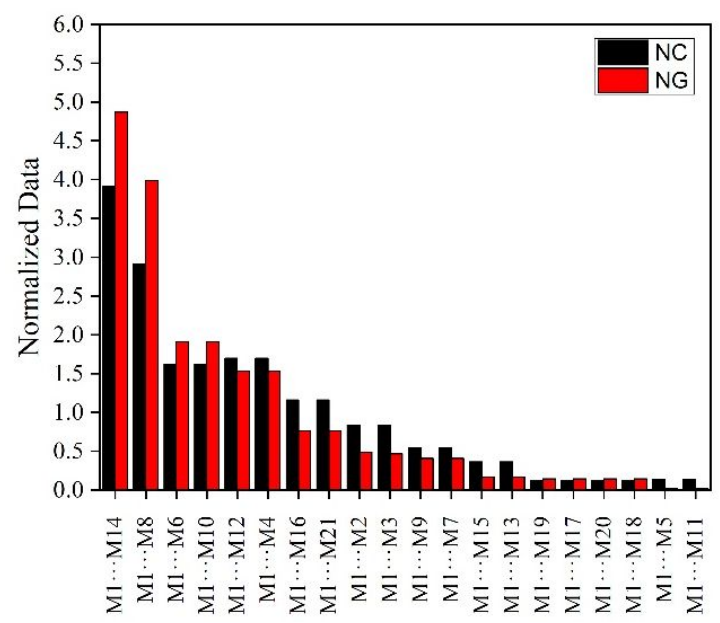

2dII

Figure S1. Topological and energetic normalized data of each dimer from the supramolecular cluster of compounds $\mathbf{1 a - d}$ and $\mathbf{2 a - d .}$ 


\section{Proposed Crystallization Mechanisms}

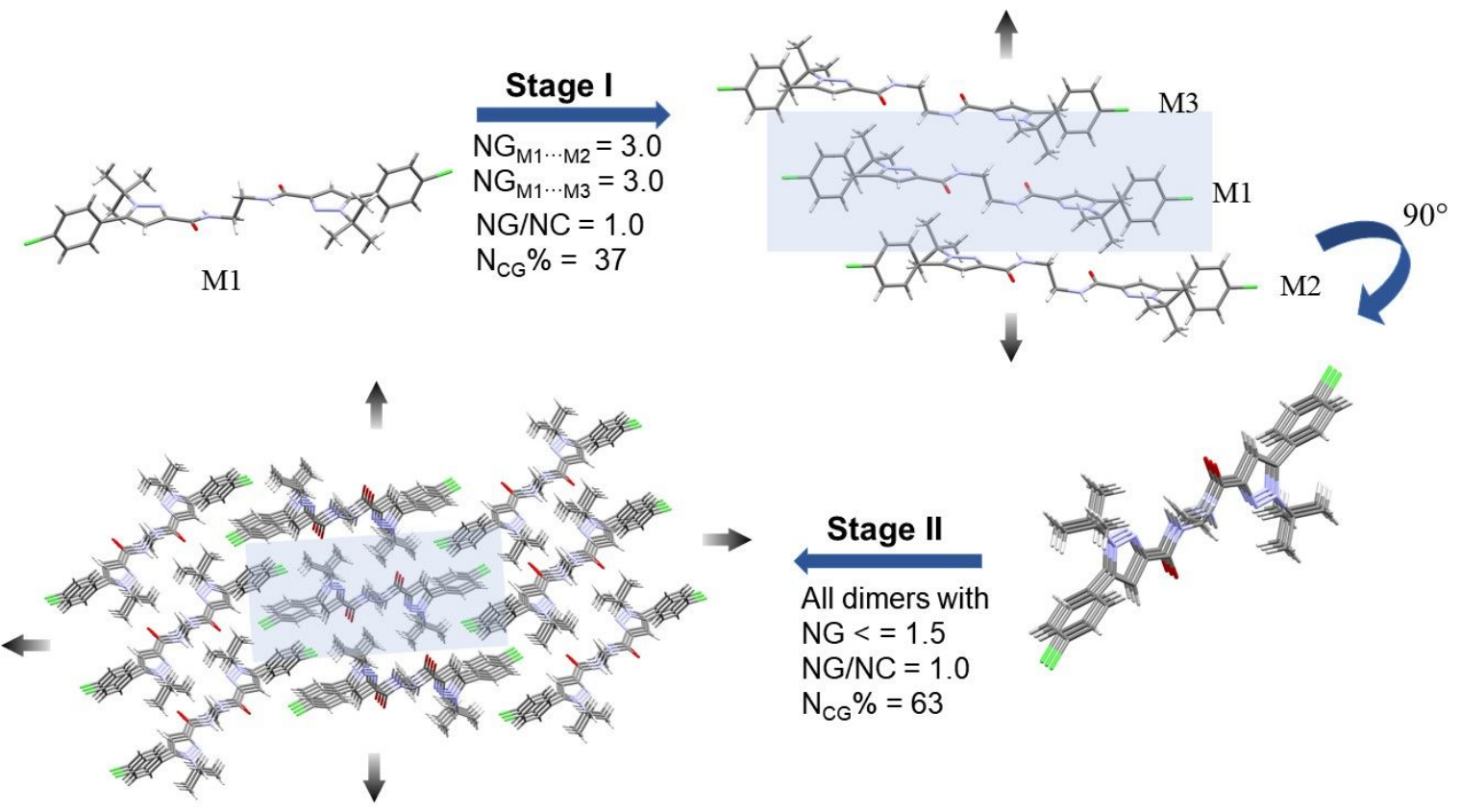

Figure S2. Proposed crystallization mechanism for polymorphs 1c. The shaded area represents the portion in the previous stage. The arrows in each stage indicate the direction of growth.

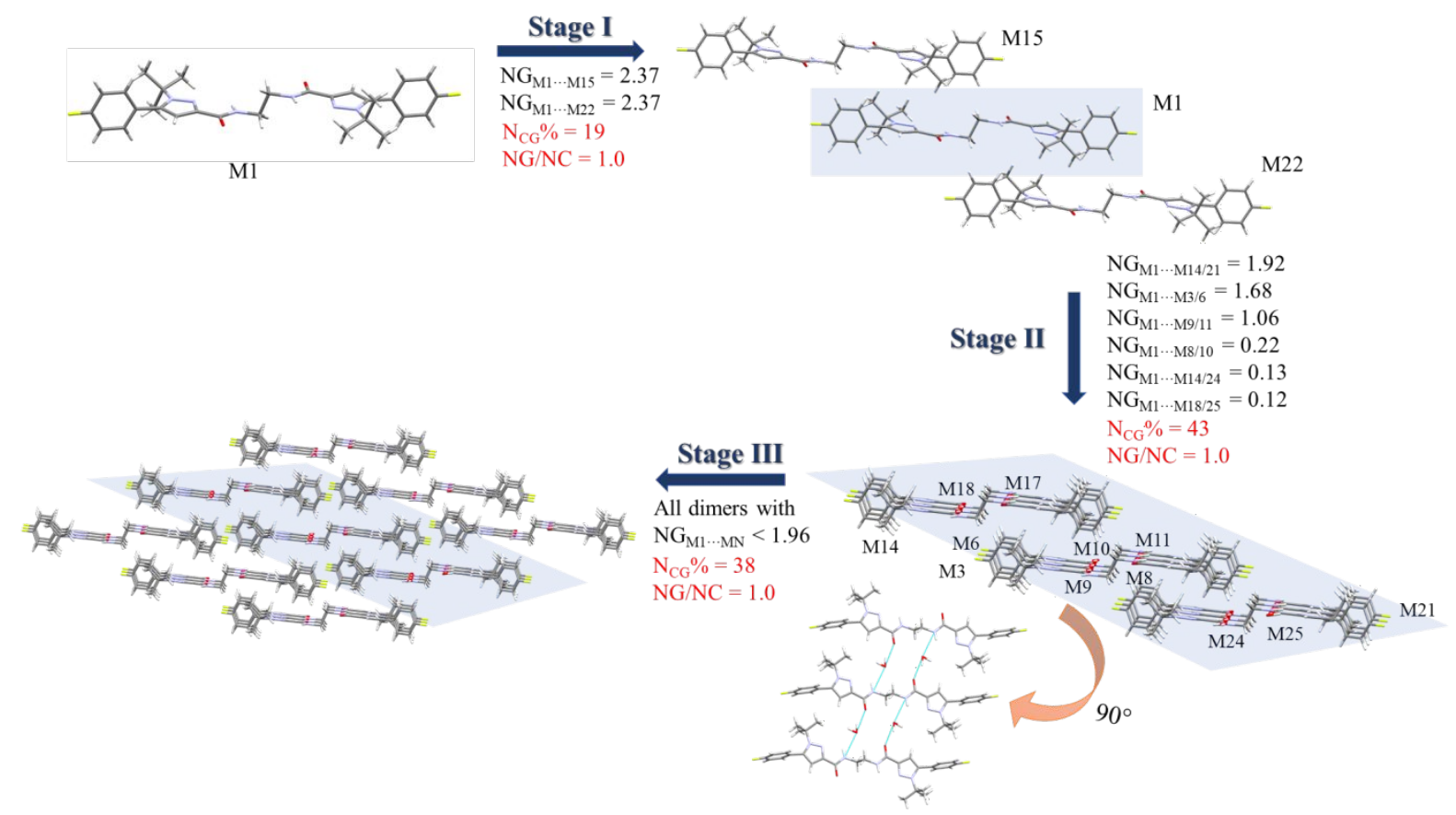

Figure S3. Proposed crystallization mechanism for polymorphs 1b. The shaded area represents the portion in the previous stage. The arrows in each stage indicate the direction of growth. 


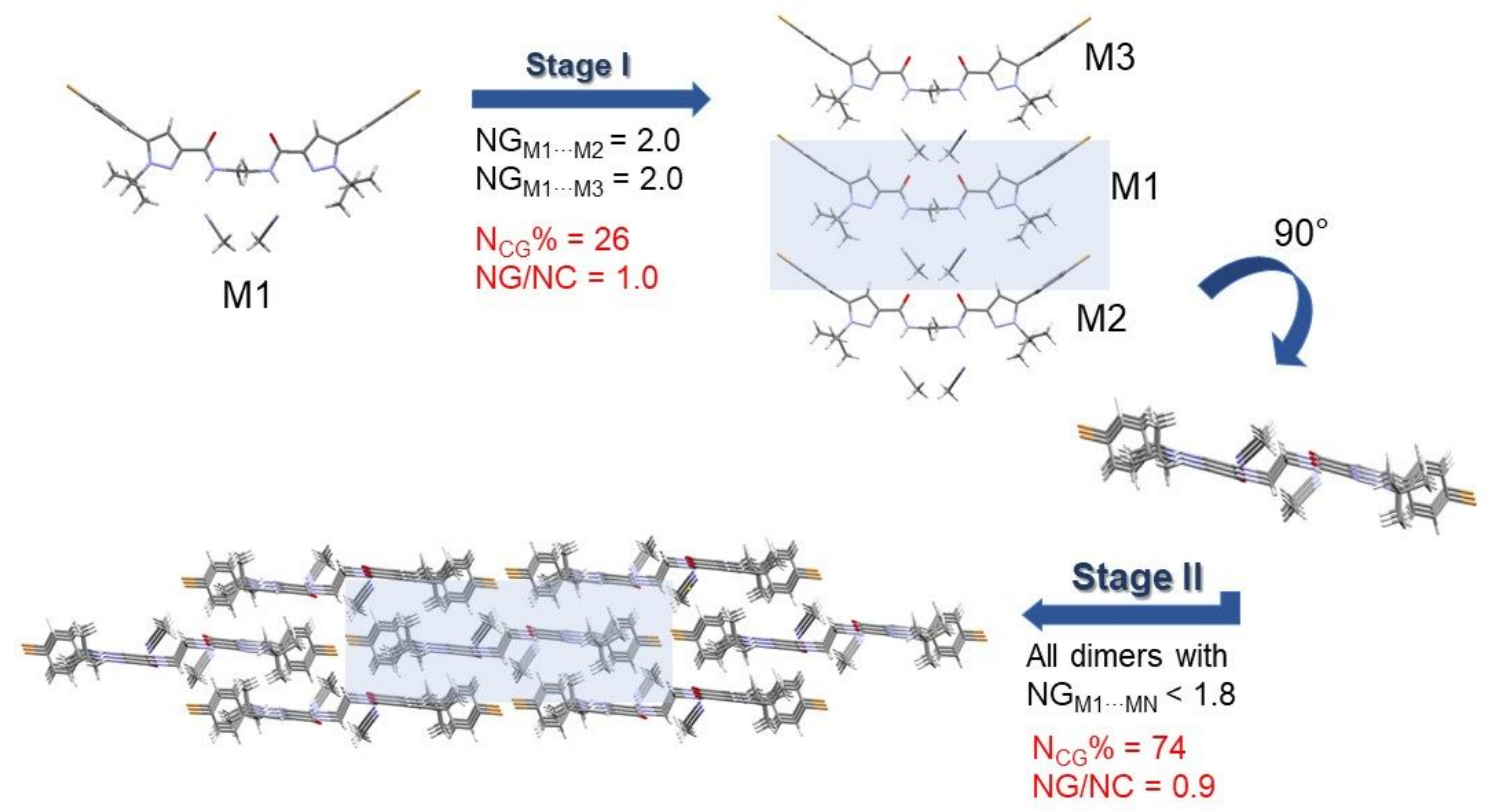

Figure S4. Proposed crystallization mechanism for polymorphs 1d. The shaded area represents the portion in the previous stage. The arrows in each stage indicate the direction of growth.
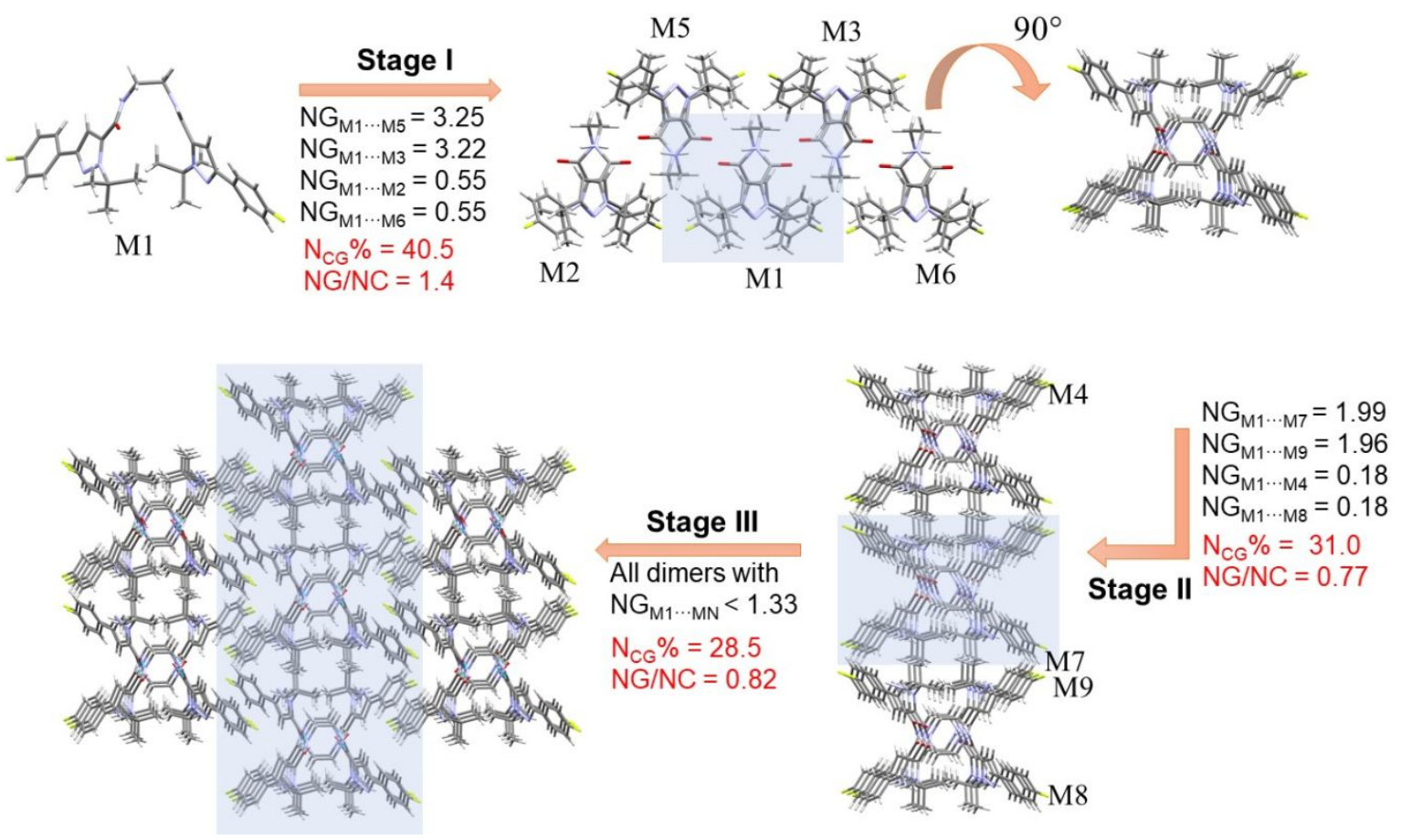

Figure S5. Proposed crystallization mechanism for polymorphs $\mathbf{2 b}$. The shaded area represents the portion in the previous stage. The arrows in each stage indicate the direction of growth. 

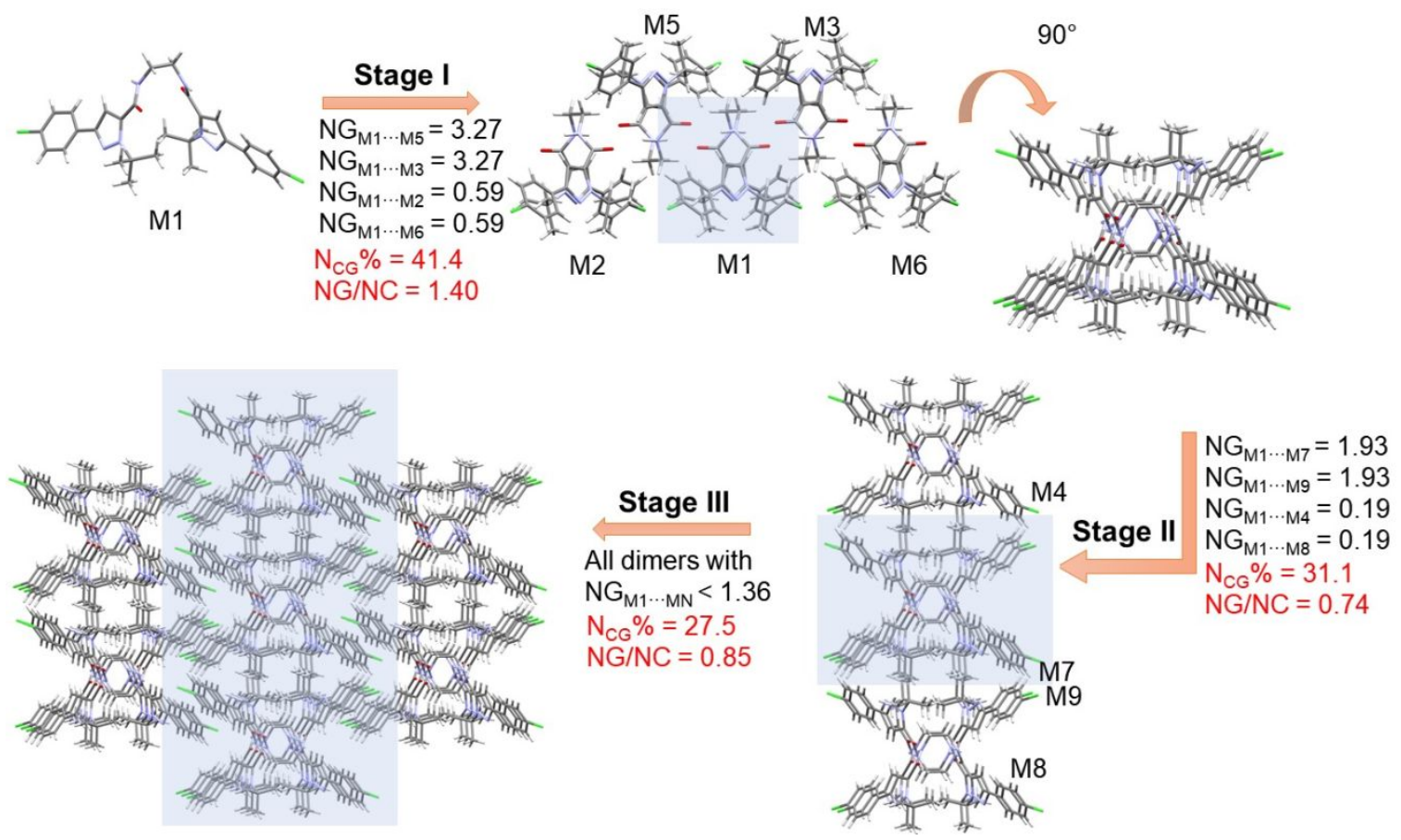

Figure S6. Proposed crystallization mechanism for polymorphs 2c. The shaded area represents the portion in the previous stage. The arrows in each stage indicate the direction of growth. 


\section{QTAIM Analysis Data}

Table S19. QTAIM data and $\mathrm{G}_{\mathrm{AI}}$ of dimers of compound $\mathbf{2 d I}$ and $\mathbf{2 d I I}$

\begin{tabular}{|c|c|c|c|c|c|c|c|c|c|c|}
\hline Dimer & Interaction & $\rho_{\mathrm{INT}}$ & $\nabla^{2} \rho$ & $\varepsilon$ & K & $\mathrm{V}$ & G & BPL & $\mathrm{H}$ & $\mathrm{G}_{\mathrm{AI}}$ \\
\hline & $0-0$ & 0.00108 & 0.00620 & 3.1685 & -0.00053 & -0.00049 & 0.00102 & 7.3805 & 0.000532 & -0.40 \\
\hline $2 \mathrm{dI}$ & $\mathrm{CH} \cdots \mathrm{N}$ & 0.00177 & 0.00696 & 0.3894 & -0.00043 & -0.00087 & 0.00131 & 6.4455 & 0.000433 & -0.65 \\
\hline M1 $\cdots M 3$ & $\mathrm{CH} \cdots \mathrm{N}$ & 0.00177 & 0.00696 & 0.3895 & -0.00043 & -0.00087 & 0.00131 & 6.4455 & 0.000433 & -0.65 \\
\hline \multirow[t]{8}{*}{ 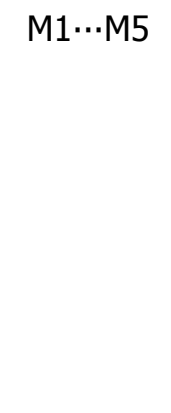 } & $\mathrm{CH} \cdots \mathrm{HC}$ & 0.00193 & 0.00842 & 1.0146 & -0.00067 & -0.00077 & 0.00144 & 5.8491 & 0.000669 & -0.71 \\
\hline & $\mathrm{CH} \cdots \mathrm{HC}$ & 0.00193 & 0.00842 & 1.0145 & -0.00067 & -0.00077 & 0.00144 & 5.8491 & 0.000669 & -0.71 \\
\hline & $\mathrm{CH} \cdots \mathrm{HC}$ & 0.00304 & 0.01340 & 0.0379 & -0.00090 & -0.00156 & 0.00245 & 5.0305 & 0.000898 & -1.12 \\
\hline & $\mathrm{CH} \cdots \mathrm{HC}$ & 0.00304 & 0.01341 & 0.0379 & -0.00090 & -0.00156 & 0.00245 & 5.0305 & 0.000898 & -1.12 \\
\hline & $\mathrm{CH} \cdots \mathrm{O}$ & 0.00783 & 0.02921 & 0.0783 & -0.00089 & -0.00553 & 0.00641 & 4.9174 & 0.000888 & -2.88 \\
\hline & $\mathrm{CH} \cdots \mathrm{O}$ & 0.00783 & 0.02921 & 0.0782 & -0.00089 & -0.00553 & 0.00642 & 4.9173 & 0.000888 & -2.88 \\
\hline & $\mathrm{NH} \cdots \mathrm{O}$ & 0.01593 & 0.06153 & 0.0483 & -0.00137 & -0.01264 & 0.01401 & 4.1072 & 0.001373 & -5.86 \\
\hline & $\mathrm{NH} \cdots \mathrm{O}$ & 0.01593 & 0.06153 & 0.0483 & -0.00137 & -0.01264 & 0.01401 & 4.1072 & 0.001373 & -5.86 \\
\hline Total & & 0.06209 & & & & & & & & -22.86 \\
\hline \multirow{14}{*}{$\begin{array}{c}\text { 2dII } \\
M 1 \cdots M 14\end{array}$} & $\mathrm{CH} \cdots \mathrm{HC}$ & 0.00262 & 0.01050 & 0.03634 & -0.00071 & -0.00122 & 0.00192 & 5.1251 & 0.00071 & -0.99 \\
\hline & $\mathrm{CH} \cdots \mathrm{HC}$ & 0.00262 & 0.01050 & 0.03634 & -0.00071 & -0.00122 & 0.00192 & 5.1251 & 0.00071 & -0.99 \\
\hline & $\mathrm{CH} \cdots \mathrm{O}$ & 0.00352 & 0.01571 & 0.62192 & -0.00100 & -0.00194 & 0.00293 & 5.8170 & 0.00100 & -1.33 \\
\hline & $\mathrm{CH} \cdots \mathrm{O}$ & 0.00352 & 0.01571 & 0.62192 & -0.00100 & -0.00194 & 0.00293 & 5.8170 & 0.00100 & -1.33 \\
\hline & $\mathrm{CH} \cdots \mathrm{HC}$ & 0.00405 & 0.01548 & 0.07514 & -0.00080 & -0.00226 & 0.00307 & 4.6925 & 0.00080 & -1.53 \\
\hline & $\mathrm{CH} \cdots \mathrm{HC}$ & 0.00405 & 0.01548 & 0.07514 & -0.00080 & -0.00226 & 0.00307 & 4.6925 & 0.00080 & -1.53 \\
\hline & $\mathrm{CH} \cdots \Pi$ & 0.00443 & 0.01533 & 3.10517 & -0.00094 & -0.00195 & 0.00289 & 6.2337 & 0.00094 & -1.67 \\
\hline & $\mathrm{CH} \cdots \Pi$ & 0.00443 & 0.01533 & 3.10517 & -0.00094 & -0.00195 & 0.00289 & 6.2337 & 0.00094 & -1.67 \\
\hline & $\mathrm{Br} \cdots n$ & 0.00502 & 0.01301 & 0.85883 & -0.00046 & -0.00234 & 0.00279 & 7.1493 & 0.00046 & -1.89 \\
\hline & $\mathrm{Br} \cdots n$ & 0.00502 & 0.01301 & 0.85883 & -0.00046 & -0.00234 & 0.00279 & 7.1493 & 0.00046 & -1.89 \\
\hline & $\mathrm{CH} \cdots \mathrm{O}$ & 0.00552 & 0.02218 & 0.10554 & -0.00088 & -0.00379 & 0.00467 & 5.1674 & 0.00088 & -2.08 \\
\hline & $\mathrm{CH} \cdots \mathrm{O}$ & 0.00552 & 0.02218 & 0.10554 & -0.00088 & -0.00379 & 0.00467 & 5.1674 & 0.00088 & -2.08 \\
\hline & $\mathrm{NH} \cdots \mathrm{O}$ & 0.01445 & 0.05547 & 0.03876 & -0.00102 & -0.01182 & 0.01284 & 4.2166 & 0.00102 & -5.45 \\
\hline & $\mathrm{NH} \cdots \mathrm{O}$ & 0.01445 & 0.05547 & 0.03876 & -0.00102 & -0.01182 & 0.01284 & 4.2166 & 0.00102 & -5.45 \\
\hline Total & & 0.07922 & & & & & & & & -29.86 \\
\hline
\end{tabular}




\begin{tabular}{|c|c|c|c|c|c|c|c|c|c|c|}
\hline & $\mathrm{CH} \cdots \Pi$ & 0.002423 & 0.008403 & 2.182752 & -0.0005 & -0.00111 & 0.001605 & 6.38494 & 0.000497 & -1.01 \\
\hline & $\mathrm{CH} \cdots \Pi$ & 0.002423 & 0.008403 & 2.182752 & -0.0005 & -0.00111 & 0.001605 & 6.38494 & 0.000497 & -1.01 \\
\hline 2dII & $\mathrm{CH} \cdots \mathrm{HC}$ & 0.002726 & 0.011354 & 0.031061 & -0.00079 & -0.00127 & 0.002054 & 5.17894 & 0.000785 & -1.13 \\
\hline \multirow[t]{7}{*}{ 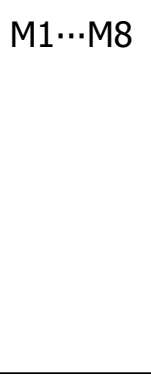 } & $\mathrm{CH} \cdots \mathrm{HC}$ & 0.002726 & 0.011354 & 0.031061 & -0.00079 & -0.00127 & 0.002054 & 5.17894 & 0.000785 & -1.13 \\
\hline & $\mathrm{CH} \cdots \mathrm{HC}$ & 0.003189 & 0.013739 & 0.715006 & -0.00099 & -0.00146 & 0.002447 & 5.47027 & 0.000987 & -1.33 \\
\hline & $\mathrm{CH} \cdots \mathrm{HC}$ & 0.003189 & 0.013739 & 0.715006 & -0.00099 & -0.00146 & 0.002447 & 5.47027 & 0.000987 & -1.33 \\
\hline & $\mathrm{CH} \cdots \mathrm{N}$ & 0.006462 & 0.020306 & 0.244956 & -0.00061 & -0.00386 & 0.004469 & 5.27269 & 0.000608 & -2.69 \\
\hline & $\mathrm{CH} \cdots \mathrm{N}$ & 0.006462 & 0.020306 & 0.244956 & -0.00061 & -0.00386 & 0.004469 & 5.27269 & 0.000608 & -2.69 \\
\hline & $\mathrm{NH} \cdots \mathrm{O}$ & 0.014583 & 0.056035 & 0.030919 & -0.00113 & -0.01174 & 0.012875 & 4.19162 & 0.001134 & -6.07 \\
\hline & $\mathrm{NH} \cdots \mathrm{O}$ & 0.014583 & 0.056035 & 0.030919 & -0.00113 & -0.01174 & 0.012875 & 4.19162 & 0.001134 & -6.07 \\
\hline Total & & 0.058766 & & & & & & & & -24.46 \\
\hline
\end{tabular}


